\title{
Nilsson-SU3 self-consistency in heavy $N=Z$ nuclei
}

\author{
A. P. Zuker, ${ }^{1}$ A. Poves,${ }^{2,3}$ F. Nowacki, ${ }^{1}$ and S. M. Lenzi ${ }^{4}$ \\ ${ }^{1}$ Université de Strasbourg, IPHC, CNRS, UMR7178, 23 rue du Loess 67037 Strasbourg, France \\ ${ }^{2}$ Departamento de Física Teórica e IFT-UAM/CSIC, Universidad Autónoma de Madrid, 28049 Madrid, Spain \\ ${ }^{3}$ ISOLDE, CERN, CH-1211 Genève, Switzerland \\ ${ }^{4}$ Dipartimento di Fisica e Astronomia dell'Università and INFN, Sezione di Padova, I-35131 Padova, Italy \\ (Received 29 April 2014; revised manuscript received 25 June 2015; published 26 August 2015)
}

\begin{abstract}
It is argued that there exist natural shell-model spaces optimally adapted to the operation of two variants of Elliott's SU3 symmetry that provide accurate predictions of quadrupole moments of deformed states. A self-consistent Nilsson-like calculation describes the competition between the realistic quadrupole force and the central field, indicating a remarkable stability of the quadrupole moments-which remain close to their quasiand pseudo-SU3 values - as the single-particle splittings increase. A detailed study of the $N=Z$ even nuclei from ${ }^{56} \mathrm{Ni}$ to ${ }^{96} \mathrm{Cd}$ reveals that the region of prolate deformation is bounded by a pair of transitional nuclei ${ }^{72} \mathrm{Kr}$ and ${ }^{84} \mathrm{Mo}$ in which prolate ground-state bands are predicted to dominate, though coexisting with oblate ones.
\end{abstract}

DOI: 10.1103/PhysRevC.92.024320

PACS number(s): 21.10.Re, 21.60.Ev, 21.60.Cs, 27.50.+e

\section{INTRODUCTION}

Large-scale shell model (LSSM) calculations, when numerically feasible, are the spectroscopic tool of choice in theoretical nuclear structure. When they are not doable it is often advised to switch to other-basically mean-fieldmethods. A common feature of these approaches is the reliance on quadrupole degrees of freedom as the backbone of nuclear structure, which in shell-model language translates as dominance of the quadrupole force, which is indeed (or should be) a classic view. Our task is to find ways to put to good use this dominance. It starts by discovering which are the model spaces in which to operate. The choice turns out to be quite unique (the extended extruder-intruder spaces to be defined soon). Though most often it leads to intractably large diagonalizations, it also happens to be tailored to take full advantage of two variantspseudo- and quasi-SU3 - of Elliott's SU3 symmetry [1]. After explaining in detail how these symmetries operate, we turn to quantitative estimates of their reliability by defining and implementing a self-consistent Nilsson [2] approach in which the interplay of a realistic quadrupole interaction with the spherical central field establishes the resilience of the predicted quadrupole moments. The controlling parameters are the quadrupole moments themselves, which, in the absence of a central field, reduce to one of their SU3-like guises.

These ideas are applied to the heavy even $N=Z$ nuclei shedding light on the hitherto poorly understood competition between prolate and oblate quadrupole coherence. In this region the full interplay of quasi- and pseudo-SU3 schemes operates, illustrating what will become the rule for welldeformed nuclei, so far only schematically explored at the onset of rotational motion at $N=90$ [3].

\section{THE NATURAL ZBM (OR EEI) MODEL SPACES}

The usual lore about shell-model spaces is that for light and medium nuclei they involve one major oscillator (HO) shell bounded by magic numbers at $N, Z=4,8,20$, and 40 , while for heavier systems the spin-orbit ( $\mathrm{SO}$ ) force takes over and the magic boundaries move to $N, Z=28,50,82$, and 126 . This view has some merit but misses two crucial points: (a) The observed shell evolution is not driven by the SO terms present in the $N N$ interactions, but by three-body forces (a word on this later); (b) the correct model spaces are larger than those defined by the SO boundaries. Let us examine the possible examples.

In the $p$ shell starting at ${ }^{4} \mathrm{He}$, as particles are added the largest orbit $p_{3 / 2}$ is "extruded" (or ejected or expelled) from the space by becoming a "closed shell" when filled, while the largest orbit in the next shell "intrudes" so as to define the first of the "EI" spaces $p_{1 / 2} d_{5 / 2} \equiv r_{1} d$ (closing at ${ }^{28} \mathrm{Si}$ ). The notation $r_{p}$ stands for "rest of the major shell of principal quantum number $p$," i.e., all the orbits except the largest one. What we miss here is that the $d_{5 / 2}$ intruder does not come alone but with an $s_{1 / 2}$ partner, as made evident by the spectrum of ${ }^{13} \mathrm{C}$ [4]. Therefore, the correct space is the first of the extended EI spaces: $r_{1} d s$ (EEI1 or ZBM [5]), with $d s=d_{5 / 2} s_{1 / 2}$, which is the first instance of a " $\Delta j=2$ " sequence.

Notation. The full harmonic oscillator shells are called $s d, p f, s d g, \ldots$, while the reverse order $d s, f p, g d s, \ldots$ is used for the $\Delta j=2$ sequences.

The next candidate comes from the $s d$ shell starting at ${ }^{16} \mathrm{O}$, where, as it fills, $d_{5 / 2}$ is separated from its partners while drawing down the largest orbit in the next shell so as to define the EI2 space: $s_{1 / 2} d_{3 / 2} f_{7 / 2} \equiv r_{2} f$ (starting at ${ }^{28} \mathrm{Si}$ and closing at ${ }^{56} \mathrm{Ni}$ ). However, we miss again that the intruder comes with its $\Delta j=2$ partner (as seen in ${ }^{29} \mathrm{Si}$ [4]), so $r_{2} f$ becomes $r_{2} f p$ (EEI2 or ZBM2), with $f p=f_{7 / 2} p_{3 / 2}$. Then we find the space, relevant for this study, $p_{1 / 2} p_{3 / 2} f_{5 / 2} g_{9 / 2} \equiv r_{3} g$ (EI3 closing at

${ }^{100} \mathrm{Sn}$ ), which is expected to become $r_{3} g d s$ (EEI3 or ZBM3), with $g d s=g_{9 / 2} d_{5 / 2} s_{1 / 2}$. Direct experimental evidence of the presence of the $\Delta j=2$ partners is hard to obtain in this region, but abundant indirect evidence is presented in this paper.

One objection to the description above is that ${ }^{12} \mathrm{C}$ and ${ }^{28} \mathrm{Si}$ are not closed shells (though ${ }^{56} \mathrm{Ni}$ is to a good approximation). However, EI numbers at $N, Z=6,14,28,50,82$, and 126 provide good boundaries and many convincing candidates to magicity in the light nuclei ( such as ${ }^{14} \mathrm{C},{ }^{22} \mathrm{O}$, and ${ }^{34} \mathrm{Si}$ ) and the only systematic magic numbers beyond. The transition from $\mathrm{HO}$ to EI major closures demands three-body mechanisms 

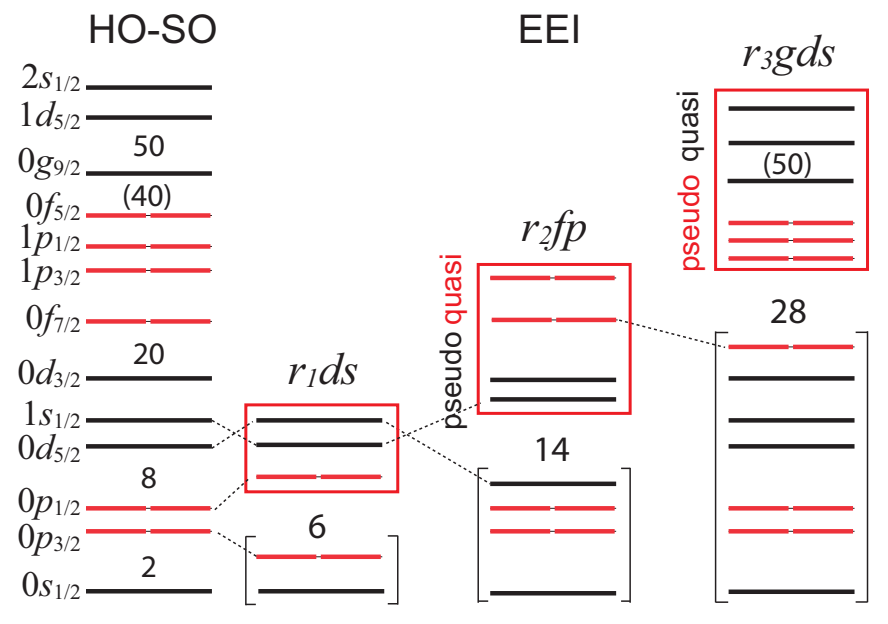

FIG. 1. (Color online) Evolution of model spaces from spin-orbit (SO) (around HO closures) to extended extruder-intruder (EEI) made of pseudo-SU3 and quasi-SU3 subspaces (explained in Sec. III).

whose irrefutable need is now established on theoretic [6] and empiric $[7,8]$ grounds. Explicit introduction of three-body forces (Fig. 3(b) vs Figs. 3(c) and 3(d) in Ref. [9]) helps but does not explain the strong magicity of ${ }^{22} \mathrm{O}$, as seen by comparing the quoted figures. The notation EI instead of the usual SO is meant to stress that the SO force-in the classic $l \cdot s$ sense-is perfectly given by existing $N N$ interactions above $\mathrm{HO}$ closures, where it is responsible for the largest orbit coming lowest [10]. However, it is definitely not responsible for the EI closures which demand splittings much larger that the $l \cdot s$ one provided by the $N N$ interactions. To fix ideas, in ${ }^{48} \mathrm{Ca}$ they would produce a $f_{7 / 2}-p_{3 / 2}$ single-particle gap equal to that in ${ }^{41} \mathrm{Ca}$, i.e., $2.5 \mathrm{MeV}$ smaller than the observed one, a discrepancy that increases to some $4.5 \mathrm{MeV}$ in ${ }^{56} \mathrm{Ni}$. The evolution of subshell $\mathrm{SO}$ ordering on top of $\mathrm{HO}$ closures to the EEI patterns is illustrated in Fig. 1 for different model spaces.

Both $r_{1} d s$ (ZBM) and $r_{2} f p$ (ZBM2 or SDFP) models lead to feasible and successful diagonalizations in the neighborhood of ${ }^{16} \mathrm{O}$ and ${ }^{40} \mathrm{Ca}[5,11]$. The $r_{3} g d s$ space is expected to work equally well around ${ }^{80} \mathrm{Zr}$-formally the magic upper boundary of the $p f$ shell-which turns out to be a splendid rotor [12]. A pure $p f$ description starts failing around $N, Z \approx$ 34 , and it could be hoped that $r_{3} g$ would cope beyond, but the calculations (always feasible though sometimes hard) fail to produce strongly deformed prolate bands demanded by the data. These are naturally explained in the $r_{3} g d s$ space, as we shall demonstrate notwithstanding the near impossibility of exact diagonalizations: first through heuristic arguments based on the approximate SU3 symmetries and then by very simple self-consistent calculations that account semiquantitatively for the interplay between the realistic quadrupole interaction and the monopole central field.

\section{QUADRUPOLE COHERENCE: SU3, PSEUDO-SU3, AND QUASI-SU3}

Nuclear rotational motion was predicted by Bohr and Mottelson in 1953 [13]. The idea was that nuclei could acquire a permanent quadrupole deformation in their intrinsic

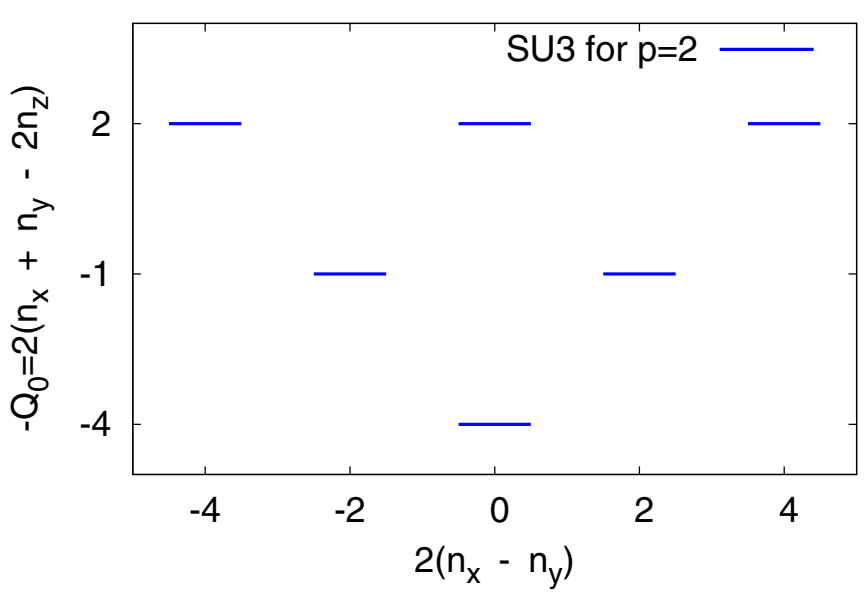

FIG. 2. (Color online) Eigenstates of $-2 q_{20}$. SU3 intrinsic states of minimum energy are obtained by orderly filling. Note that $-Q_{0}$ is plotted on the vertical axis.

frame, which would translate into a $J(J+1)$ spectrum in the laboratory frame. Historically, this first example of spontaneously broken symmetry was confronted with the need to explain how a deformed intrinsic state-which has no definite angular momentum $J$-could be an eigenstate of a system that must necessarily conserve $J$. The elegant way out was found by Elliott, whose SU3 model [1] provides a rigorous example of intrinsic states that are not eigenstates of a Hamiltonian $H$ but of $H-\lambda J(J+1)$.

More precisely, $H$ is taken to be the quadrupole force $-2 q \cdot 2 q$, with $q \equiv q^{2 m}=r^{2} C^{2 m}=r^{2} \sqrt{4 \pi / 5} Y^{2 m}$ acting in a full major HO shell. Then the eigenstates have the form $E(L, i)=E(i)+3 L(L+1)$, where $L$ is the orbital angular momentum and $E(i)$ the energy of one of the possible intrinsic states. We are interested only in those that maximize the intrinsic quadrupole moment, which we write in terms of oscillator quanta $Q_{0}=2 q^{20}=\left(2 n_{z}-n_{x}-n_{y}\right)$. Taking, for example, $p=n_{x}+n_{y}+n_{z}=2$, the six possible single-particle states $\left[n_{z} n_{x} n_{y}\right]=[200],[110],[101],[020],[011],[002]$ can be disposed as in Fig. 2. The intrinsic states are the determinants obtained by filling the fourfold degenerate orbits (two neutrons and two protons of spins up and down) from below (prolate states with $Q_{0}>0$ ) or from above (oblate states with $Q_{0}<0$ ). Prolate filling is favored as it leads to larger $\left|Q_{0}\right|$.

Originally, SU3 was expected to apply to the $s d$ shell. Indeed, the four particles in ${ }^{20} \mathrm{Ne}\left(Q_{0}=16\right)$ produce a good rotor and eight particles in ${ }^{24} \mathrm{Mg}$ - because of the degeneracy of the $Q_{0}=1$ levels in Fig. 2-lead to triaxiality, associated with the mixing of $K=0$ and $K=2$ prolate bands. For 12 particles in ${ }^{28} \mathrm{Si}$, both shapes are expected to be degenerate $\left(\left|Q_{0}\right|=24\right)$. Observation does not quite square with predictions: The $K=2$ band in ${ }^{24} \mathrm{Mg}$ is higher than expected, and the "nearly degenerate" oblate and prolate states in ${ }^{28} \mathrm{Si}$ are separated by some $6 \mathrm{MeV}$ with a third candidate coming in (the $d_{5 / 2}^{12} N=Z=14$ closure in Fig. 1). Still, the departure from strict SU3 validity should not hide the fact that ${ }^{24} \mathrm{Mg}$ has a $K=2(\gamma)$ band, and that three of the six lowest states in ${ }^{28} \mathrm{Si}$ have $J=0^{+}$, a forerunner of other spectacular coexistence situations. 
Though Elliott's conceptual breakthrough was obscured by the limited applicability of the exact SU3 symmetry, its indicative value remains high, as illustrated by examining the possible forms of the $q^{20}$ operator in $L S$ and $j j$ formalisms in Eqs. (1)-(5): They will be seen to suggest naturally the pseudo- and quasi-SU3 variants that are the backbone of a full shell-model description of rotational motion:

$$
\begin{aligned}
\left\langle p l\left|r^{2}\right| p l\right\rangle & =p+3 / 2, \\
\left\langle p l\left|r^{2}\right| p l+2\right\rangle & =-[(p-l)(p+l+3)]^{1 / 2}, \\
\left\langle l m\left|C_{2}\right| l m\right\rangle & =\frac{l(l+1)-3 m^{2}}{(2 l+3)(2 l-1)},\left\langle l m\left|C_{2}\right| l+2 m\right\rangle \\
& =\frac{3}{2}\left\{\frac{\left[(l+2)^{2}-m^{2}\right]\left[(l+1)^{2}-m^{2}\right]}{(2 l+5)(2 l+3)^{2}(2 l+1)}\right\}^{1 / 2}, \\
\left\langle j m\left|C_{2}\right| j m\right\rangle & =\frac{j(j+1)-3 m^{2}}{2 j(2 j+2)},\left\langle j m\left|C_{2}\right| j+2 m\right\rangle \\
& =\frac{3}{2}\left\{\frac{\left[(j+2)^{2}-m^{2}\right]\left[(j+1)^{2}-m^{2}\right]}{(2 j+2)^{2}(2 j+4)^{2}}\right\}^{1 / 2},
\end{aligned}
$$

$\left\langle j m\left|C_{2}\right| j+1 m\right\rangle=-\frac{3 m\left[(j+1)^{2}-m^{2}\right]^{1 / 2}}{j(2 j+4)(2 j+2)}$.

Intrinsic states can be constructed by diagonalizing $q^{20}$, which can be done in three possible ways, described next, after noting that, so far, we have assumed dimensionless oscillator coordinates and made no difference between $\left\langle 2 q^{20}\right\rangle$ and $Q_{0}$. Dealing with electromagnetic properties demands to recover dimensions so $r^{2} \rightarrow r^{2} b^{2}$, where $b^{2}$ is the oscillator parameter. Then $Q_{0} \rightarrow Q_{0} b^{2}$. However, $\left\langle 2 q^{20}\right\rangle$ is best kept adimensional when working with the quadrupole interaction. So now $Q_{0} / b^{2}=\left\langle 2 q^{20}\right\rangle$, and the choice of notation will depend on context.

\section{A. Strict SU3}

Use Eqs. (1)-(3) in $L S$ form to obtain exactly Fig. 2. Alternatively, use Eqs. (1), (2), (4), and (5) in $j j$ form to incorporate spin, leading to the bottom panel of Fig. 3. Only positive values of $K \equiv|m|$ are shown. Each orbit may contain two neutrons and two protons. Note that if in Fig. 2 spin is allowed each orbit splits into $2\left(n_{x}-n_{y}\right) \equiv 2 m \rightarrow 2(m \pm 1 / 2)$ and the one-to-one correspondence with the bottom panel of Fig. 3 becomes evident.

The importance of SU3 goes well beyond its mathematical elegance: It rests on the introduction of the $q \cdot q$ interaction restricted to a single major $\mathrm{HO}$ shell, which, as demonstrated in Ref. [14], is the major collective ingredient of realistic Hamiltonians (i.e., consistent with two nucleon data).

\section{B. Pseudo-SU3}

Pseudo-SU3 [15] is adapted to $r_{p}$ spaces whose orbits have the same angular momentum $j$ sequences as those of full HO major shell with total quantum number $p-1$ and proceeds as if $r_{p} \equiv \mathrm{HO}(p-1)$, in our case $r_{3} \equiv s d$. For the angular
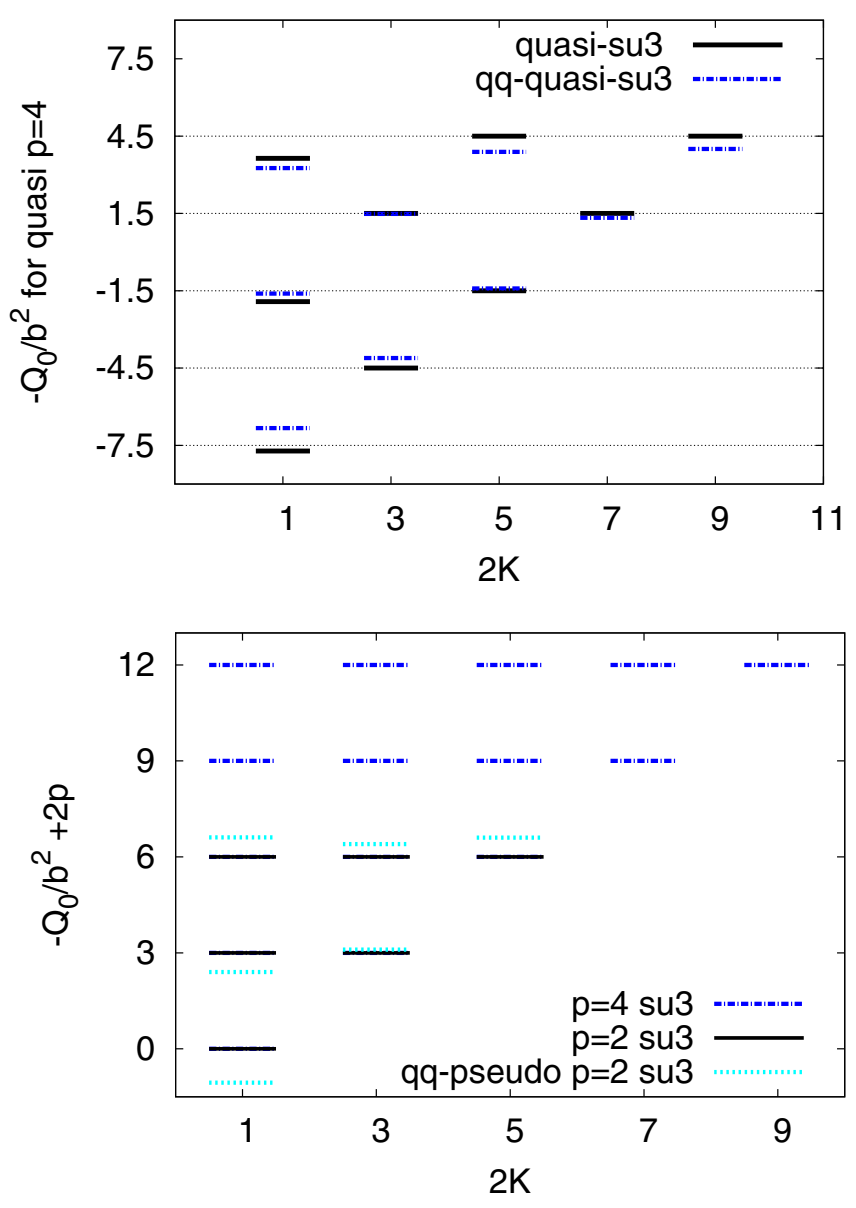

FIG. 3. (Color online) The Zuker-Retamosa-Poves (ZRP) diagrams. (Top) Intrinsic states in the $p=4 g d s$ space for the quasi-SU3 model and for the exact $q \cdot q$ calculation. See text for explanation of thin lines. (Bottom) Intrinsic states of SU3 or pseudo-SU3 for $p=2$ and 4 . For the former, exact $q \cdot q$ values ("qq-pseudo") are also shown. Each orbit may contain two neutrons and two protons. The lowest six orbits are common to $p=2$ and 4 .

Eqs. (4) and (5) the identity is perfect but the radial Eqs. (1) and (2) raise a problem: $r_{3}$ has $p=3$ and $s d$ has $p=2$. The bottom panel of Fig. 3 exhibits both of the strict SU3 (or pseudo-SU3) values for $p=2$ and 4 , as well as the exact result of diagonalizing $2 q^{20}$ in the $r_{3}$ space, collected under p-d in Table I. It is seen that the differences are substantial, but they do not invalidate the existence of an underlying SU3 symmetry: The $q \cdot q$ interactions in the $s d$ and $r_{3}$ spaces are very different, but their behavior is qualitatively similar. In what follows we always use the exact $r_{3}$ variant of $q \cdot q$.

\section{Quasi-SU3}

Quasi-SU3 [3,16] is adapted to $\Delta j=2$ spaces. Then $\left\langle j m\left|C_{2}\right| j+1 m\right\rangle$ in Eq. (5) plays no role. Now identify the $\Delta j=2$ sequence to a $\Delta l=2$ one. In our case it is $J=$ $9 / 2,5 / 2,1 / 2$ to $l=4,2,0$. Then replace Eqs. (1), (2), and (4) with Eqs. (1), (2), and (3), through $l \rightarrow j, p \rightarrow p+1 / 2$, $m \rightarrow m+1 / 2$, and $-m \rightarrow-m-1 / 2:(m>0)$. This defines a quasi- $q_{20}$ operator whose spectrum is shown (under "quasi- 
TABLE I. Eigenvalues of $-2 q^{20}$ for the $i$ th quasi- $g d s$ (denoted q) and pseudo- $r_{3}$ (denoted p) orbits; q-s, p-s are the results using the schematic quasi- and pseudoquadrupole forces, respectively, in Fig. 3. q-d and p-d are the results of diagonalizing the exact quadrupole interaction; c-q and c-p are the corresponding cumulated absolute values for $n$ particles in units of $b^{2}$.

\begin{tabular}{|c|c|c|c|c|c|c|c|c|c|}
\hline$i$ & 1 & 2 & 3 & 4 & 5 & 6 & 7 & 8 & 9 \\
\hline q-s & -7.71 & -4.50 & -1.92 & -1.50 & 1.50 & 1.50 & 3.64 & 4.50 & 4.50 \\
\hline$q-d$ & -6.83 & -4.11 & -1.61 & -1.42 & 1.33 & 1.48 & 3.26 & 3.90 & 4.00 \\
\hline p-s & -4.00 & -1.00 & -1.00 & 2.00 & 2.00 & 2.00 & & & \\
\hline $\mathrm{p}-\mathrm{d}$ & -5.06 & -1.41 & -1.08 & 2.37 & 2.57 & 2.61 & & & \\
\hline \multicolumn{10}{|c|}{$Q_{0}$ values for $n$ particles (c-q for $g d s$ and c-p for $r_{3}$ ) } \\
\hline$c-p$ & 20.24 & 25.88 & 30.20 & 20.72 & 10.44 & 0.00 & & & \\
\hline
\end{tabular}

su3") in the top panel of Fig. 3, where thin lines indicate a one-to-one correspondence with Fig. 2, with bandheads at $2 p-1 / 2$, except for $K=1 / 2$ for even $p$. For odd $p$ the correspondence is perfect throughout. The spectrum for the genuine $q^{20}$ operator ("qq-quasi-su3" in the figure) is seen to be quite close to the schematic one (numerical values are collected under q-d in Table I).

Table I compares the schematic orbits of Fig. 3 with the ones obtained by diagonalizing $2 q^{20}$ associated with "true" $2 q \cdot 2 q$ and not one of its variants. The two bottom lines give the cumulated values after filling up to $i$ th orbit with two neutrons and two protons. Thus, for 12 particles in $r_{3}$ and 4 in $g d s$ we find $\left\langle 2 q^{20}\right\rangle=30.20+27.32=50.52$. This table is the relevant one for prolate states.

Quasi-SU3 strongly prefers prolate solutions, as Fig. 3 makes clear: It is more advantageous to fill orbits from below than from above.

\section{Single-orbit quadrupole}

When the $g$ orbit becomes sufficiently depressed with respect to its $d s$ partners their influence can be neglected and we move to the single $j$ orbit regime with quadrupole moments given by

$$
Q_{0}=2\left\langle r^{2} C_{2}\right\rangle=\sum_{m}(p+3 / 2) \frac{j(j+1)-3 m^{2}}{2 j(j+1)},
$$

which shows that, before midshell, filling large $m$ values (negative $Q_{0}$ ) is favored. The situation is reversed after midshell. Though the notion of shape is questionable in this case, states with positive and negative $Q_{0}$ are referred to as prolate and oblate, respectively.
Table II collects the possible values of $\left\langle 2 q^{20}\right\rangle$ for the $g_{9 / 2}$ orbit and the $r_{3}$ space, where one may wish to speak in terms of holes rather than particles, and the table allows for all possibilities. For example, under $\mu=8$ we find that $\left\langle 2 q^{20}\right\rangle=25.88$ for prolate particles, 20.72 for prolate holes, -25.88 for oblate holes, and -20.72 for oblate particles.

To guarantee a bona fide intrinsic state, $Q_{0}$ must coincide with the values extracted either from the spectroscopic quadrupole moment $\left(Q_{0 s}\right)$,

$$
\begin{aligned}
Q_{\text {spec }}(J) & =\left\langle J J\left|3 z^{2}-r^{2}\right| J J\right\rangle, \\
Q_{0 s} & =\frac{(J+1)(2 J+3)}{3 K^{2}-J(J+1)} Q_{\text {spec }}(J), \quad K \neq 1,
\end{aligned}
$$

for Bohr Mottelson rotors or the corresponding $B(E 2)$ transitions $\left(Q_{0 t}\right)$,

$$
\begin{aligned}
B(E 2, J \rightarrow J-2)= & \frac{5}{16 \pi} e^{2}|\langle J K 20 \mid J-2, K\rangle|^{2} Q_{0 t}^{2}, \\
& K \neq 1 / 2,1
\end{aligned}
$$

The condition $Q_{0} \approx Q_{0 s} \approx Q_{0 t}$ is well fulfilled by SU3 states and its variants. ( $Q_{0 s}$ may be tricky, though, as it is more sensitive to details than $Q_{0 t}$. For an example, refer to Sec. VA2.)

\section{COMPUTATIONAL STRATEGY: SU3-NILSSON SELF-CONSISTENCY}

The guiding idea is that once quadrupole dominance sets in, the wave functions are basically given by the quadrupole force, which is quite immune to single-particle details. In other

TABLE II. (Top) Intrinsic prolate and oblate quadrupole moments $\left\langle 2 q^{20}\right\rangle$ for $v$ particles in the $0 g_{9 / 2}$ orbit $(N=Z)$. (Bottom) Pseudo-SU3 $\left\langle 2 q^{20}\right\rangle$ for $\mu$ prolate particles (p-p) or $\mu$ prolate holes (p-h), $-\left\langle 2 q^{20}\right\rangle$ for $\mu$ oblate particles [-(o-p)] or $\mu$ oblate holes [-(o-h)].

\begin{tabular}{lcccccccc}
\hline \hline$v$ & 2 & 4 & 6 & 8 & 10 & 12 & 14 \\
\hline prol & 5.33 & 10.66 & 14.66 & 18.66 & 20 & 21.33 & 18.66 \\
-obl & 8 & 16 & 18.66 & 21.33 & 20 & 18.66 & 14.66 \\
$\mu$ & 2 & 4 & 6 & 8 & 10 & 12 & 10 \\
\hline p-p; -(o-h) & 10.12 & 20.24 & 23.04 & 25.88 & 28.05 & 30.20 & 25.46 \\
p-h; -(o-p) & 5.22 & 10.44 & 15.66 & 20.72 & 25.46 & 30.20 & 28.04 \\
\hline \hline
\end{tabular}


words, $\left\langle 2 q^{20}\right\rangle$ varies little. Our aim is to estimate $\left\langle 2 q^{20}\right\rangle$ and understand the reason for its stability.

We are interested in even $N=Z=28$ to 48 nuclei. Full $p f$ diagonalizations are possible, but their interest is restricted to the lightest species. For $r_{3} g$ exact calculations are also possible that account for oblate states. The JUN45 interaction [17] is used throughout the region. Though the $r_{3} g$ space is of limited relevance, the exact calculations serve as a test of our simple models. For the more collective prolate states the full $r_{3} g d s$ space is necessary and exact calculations are not presently feasible, so we introduce a self-consistent version of Nilsson's model that reduces to quasi- and pseudo-SU3 in the absence of a central field [18].

\section{A. Example of naive $B E 2$ estimate}

For SU3 the correct value of $Q_{0}$ to be used in Eqs. (7) and (8) is $Q_{0}=\left(\left\langle 2 q^{20}\right\rangle+3\right) b^{2}[1,19]$ with $\left\langle 2 q^{20}\right\rangle$ given in Table I or II. In what follows we adopt this form in all cases.

The procedure is simple: Use the tables to match oblate pseudo-SU3 states in $r_{3}$ to oblate states in $g$ and prolate pseudo-SU3 states in $r_{3}$ to prolate quasi-SU3 states in $g d s$. For instance, choose 16 particles and decide that we are interested in ${ }^{72} \mathrm{Kr}$ configurations with 12 particles in $r_{3}$ and 4 above. From the tables we have for $\left\langle 2 q^{20}\right\rangle$ the following possibilities:

$$
\begin{aligned}
& \text { Oblate } \\
& \left\langle 2 q^{20}\right\rangle=-30.2 \text { for } m=12 \text { in pseudo, } \\
& \left\langle 2 q^{20}\right\rangle=-16 \text { for } n=4 \text { in } g, \\
& \text { Total } Q_{0} / b^{2}=-(30.2+3+16)=-49.2 \\
& \text { Prolate } \\
& \left\langle 2 q^{20}\right\rangle=30.2 \text { for } m=12 \text { in pseudo, } \\
& \left\langle 2 q^{20}\right\rangle=27.32 \text { for } n=4 \text { in quasi, } \\
& \text { Total } Q_{0} / b^{2}=30.2+3+27.32=60.52
\end{aligned}
$$

Recover dimensions through $b^{2} \approx 41.4 / \hbar \omega \mathrm{fm}^{2}, \quad \hbar \omega=$ $45 A^{-1 / 3}-25 A^{-2 / 3}$.

Now assume a conventional $2 \hbar \omega$ scalar effective charge, $e_{0}=e_{\nu}+e_{\pi}=2$ chosen throughout in what follows. Then, for $A=72, b^{2}=4.42 e \mathrm{fm}^{2}$, we have $Q_{0} \approx$ $-217 e \mathrm{fm}^{2}$ (oblate) and $267 e \mathrm{fm}^{2}$ (prolate).

The $2 \hbar \omega$ effective charge is caused by coupling states in a major HO shell to the giant quadrupole resonance. A rigorous derivation leads $e_{0}=1.77$ [14], a number to be preferred [20], and shown in parentheses below. Using $B\left(E 2: 2^{+} \rightarrow 0^{+}\right)=$ $\left[Q_{0}\right]^{2} / 50.3$ from Eq. (8) leads to

$B\left(E 2: 2^{+} \rightarrow 0^{+}\right) \approx 936(725) e^{2} \mathrm{fm}^{4}$ for oblate;

$B\left(E 2: 2^{+} \rightarrow 0^{+}\right) \approx 1422(1101) e^{2} \mathrm{fm}^{4}$ for prolate.

When working in EI or EEI spaces it becomes necessary to account for $0 \hbar \omega$ polarization effects, in our case owing to coupling to the lowest $J=2^{+}$state in ${ }^{56} \mathrm{Ni}$. The effect is estimated later, leading to $e_{0} \gtrsim 2$.

\section{B. Nilsson revisited: The $\mathrm{MZ}$ equations}

The estimates above neglect single-particle effects. To account for them demands solving the Schrödinger equation for the quadrupole force in the presence of a central field, a task as hard as the general problem. In Ref. [16] Martínez-Pinedo and Zuker (MZ) proposed to reduce it, by linearization, to a Nilsson-type Hamiltonian. That this should be possible seems obvious, but the implementation is not trivial. Because of a subtlety that was missed at the time, the project was left unfinished. We retake it.

We would like to solve

$$
\begin{gathered}
H_{m q}=\sum \epsilon_{i} n_{i}-\hbar \omega \kappa\left(\frac{2 q_{p}}{\mathcal{N}_{2 q, p}}+\frac{2 q_{p+1}}{\mathcal{N}_{2 q, p+1}}\right)^{2}, \\
\mathcal{N}_{2 q, p}^{2}=\sum\left(2 q_{r s}\right)^{2}=\frac{5}{2} \sum_{k=0}^{p}(k+1)(2 p-3 k)^{2},
\end{gathered}
$$

where we have borrowed from Ref. [14] the normalized form of the quadrupole force that emerges naturally when it is extracted from a realistic interaction $\left(q_{p}\right.$ is the quadrupole operator in major shell $p$; the square stands for scalar product). This form ensures that $\kappa \approx 0.22-0.25$ is a universal constant that demands a $30 \%$ renormalization owing to coupling to the $2 \hbar \omega$ quadrupole degrees of freedom [14]. It also ensures that nuclei do not become needles, thus solving the crippling problem of the naive quadrupole force [21]. In all that follows we have fixed $\kappa=0.3$.

To prepare for linearization replace $q$ with $q_{20}$ operators (notice that we use sometimes $q_{20}$ instead of $q^{20}$ for typographical reasons),

$$
\begin{aligned}
H_{m q 0} & =\sum \varepsilon_{i} n_{i}-\hbar \omega \kappa\left(\frac{2 q_{20, p}}{\mathcal{N}_{2 q_{20, p}}}+\frac{2 q_{20, p+1}}{\mathcal{N}_{2 q_{20, p+1}}}\right)^{2}, \\
\mathcal{N}_{2 q_{20, p}}^{2} & =\sum\left(2 q_{20, r s}\right)^{2}=\sum_{k=0}^{p}(k+1)(2 p-3 k)^{2} .
\end{aligned}
$$

Note that Eq. (12) is obtained by summing the squares of the levels in Fig. 2.

Now concentrate on a single space. The operation amounts to replacing $2 q \cdot 2 q$ with $2 q_{20} 2 q_{20}$ and demands some care because $q_{20}$ is a sum of neutron and proton contributions $q_{20}=$ $q_{20}^{\nu}+q_{20}^{\pi}$. As calculations are done for each fluid separately, the correct linearization for the neutron operators, say, is

$$
q_{20} q_{20} \rightarrow q_{20}^{v}\left\langle q_{20}^{\nu}+2 q_{20}^{\pi}\right\rangle \approx 3 q_{20}^{\nu}\left\langle q_{20}^{\nu}\right\rangle \text { if }\left\langle q_{20}^{\nu}\right\rangle \approx\left\langle q_{20}^{\pi}\right\rangle,
$$

leading to the $\mathrm{MZ}$ equation

$$
H_{m q 0}=\sum \varepsilon_{i} n_{i}-\frac{3 \hbar \omega \kappa}{\mathcal{N}_{2 q_{20}, p}^{2}}\left\langle 2 q_{20}^{\nu}\right\rangle 2 q_{20}^{\nu} .
$$

The subtlety missed in Ref. [16] was the need to change $\mathcal{N}_{2 q, p}$ into $\mathcal{N}_{2 q_{20}, p}$ in going from Eqs. (9) to (11), thus making it impossible to discover the proper way to proceed, which now can be implemented [18].

To find the proper generalization of Eq. (13), note that in the full space $q$ becomes a sum of four contributions $q_{20}=q_{20}^{v u}+$ $q_{20}^{\pi u}+q_{20}^{v d}+q_{20}^{\pi d}\left(u=g d s, d=r_{3}\right)$. Repeating the arguments leading to Eq. (13) and setting $\mathcal{N}_{i}=\mathcal{N}_{2 q_{20}, i}$ leads to the general 


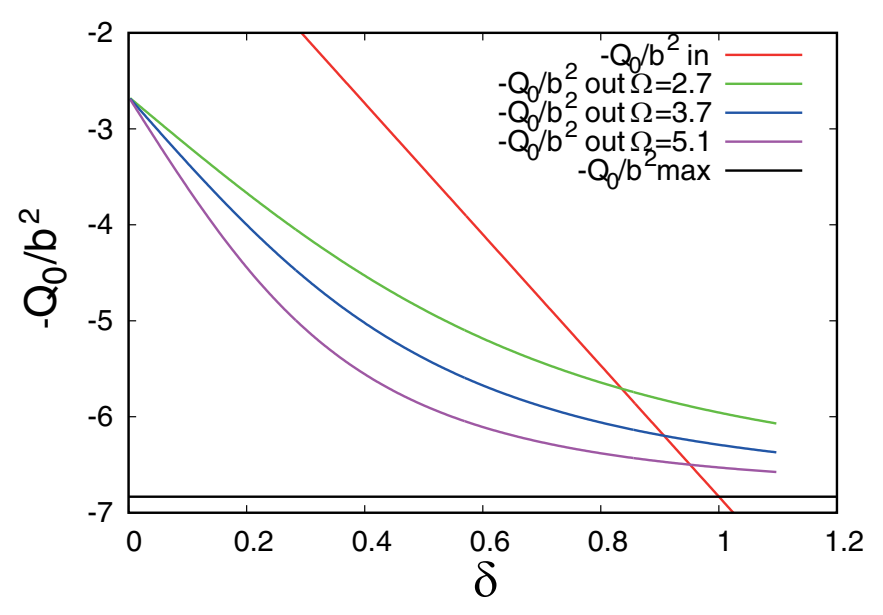

FIG. 4. (Color online) Calculation of $\left\langle 2 q_{20}\right\rangle$ in $(g d s)^{4}$. Input values are in red. Output values were obtained solving Eq. (13) for $\varepsilon_{i}=0,1,2 \mathrm{MeV}$ for $i=g, d, s$, respectively. When input and output values coincide, self-consistency is achieved, i.e., when lines cross, which happens at abscissae $0.83,0.91,0.95$ for $\Omega=\hbar \omega \kappa=$ 2.7,3.7,5.1, respectively. $\delta=0\left\langle 2 q^{20}\right\rangle=2.666$ corresponds to one prolate $g$ orbit (from Table II).

MZ equation

$$
\begin{gathered}
H_{\mathrm{sp}}-4 \hbar \omega \kappa \frac{q_{4}^{v}}{\mathcal{N}_{4}^{2}}\left(\left\langle q_{4}^{v}\right\rangle+\left\langle 2 q_{4}^{\pi}\right\rangle+\left\langle 2 q_{3}^{v}\right\rangle \frac{\mathcal{N}_{4}}{\mathcal{N}_{3}}+\left\langle 2 q_{3}^{\pi}\right\rangle \frac{\mathcal{N}_{4}}{\mathcal{N}_{3}}\right) \\
\approx H_{\mathrm{sp}}-4 \hbar \omega \kappa \frac{q_{4}^{v}}{\mathcal{N}_{4}^{2}}\left(3\left\langle q_{4}^{v}\right\rangle+6\left\langle q_{3}^{v}\right\rangle\right) \\
=H_{\mathrm{sp}}-\beta \hbar \omega \kappa \frac{\left\langle 2 q_{4}^{v}\right\rangle}{\mathcal{N}_{4}^{2}} 2 q_{4}^{v}
\end{gathered}
$$

where we have introduced a boost factor $\beta$, set $q_{i}^{v}=$ $q_{i}^{\pi}$, and used the correct numbers from Eq. (12), $\mathcal{N}_{2 q, 3}=$ $\sqrt{90 \times 2.5} \approx 15, \mathcal{N}_{2 q, 4}=\sqrt{210 \times 2.5} \approx 23$, to approximate $\mathcal{N}_{4} / \mathcal{N}_{3}=22.91 / 15 \approx 1.5$.

As the $\left\langle 2 q^{20}\right\rangle$ ranges will be $\left\langle 2 q_{3}\right\rangle \approx 30$ and $\left\langle 2 q_{4}\right\rangle=27-55$, the modest value of $\beta=3$ in Eq. (13) will increase to about $\beta=9-12$, but the work involved in solving Eqs. (13) and (14) is identical.

Let us examine the steps involved.

(a) Equation (13) is solved setting as inputs $\left\langle 2 q_{20 \text { in }}\right\rangle=$ $\delta\left\langle 2 q_{20 \max }\right\rangle$, which for $\delta=1$ yields the maximum value of $\left\langle 2 q_{20}\right\rangle$ (the one obtained at $\varepsilon_{i}=0$ ). The resulting eigenvalue can be written as

$$
E(\delta)=\left\langle H_{\mathrm{sp}}\right\rangle-\frac{3 \hbar \omega \kappa}{\mathcal{N}_{2 q_{20}}^{2}} \delta\left\langle 2 q_{20 \max }\right\rangle\left\langle 2 q_{20 \text { out }}\right\rangle . x
$$

(b) Extract $\left\langle 2 q_{20 \text { out }}\right\rangle$, use it as the next input, and iterate until $\left\langle 2 q_{20 \text { in }}\right\rangle=\left\langle 2 q_{20 \text { out }}\right\rangle$. Figure 4 sums up the procedure.

(c) Guess the energies. The comparison of the resulting $\left\langle 2 q_{20 \text { out }}\right\rangle=Q_{0} / b^{2}$ with exact results turns out to be systematically satisfactory. Some examples are given in Sec. IV C. As a reasonable estimate of $Q_{0} / b^{2}$ amounts to a good guess of intrinsic state from which the energy could be extracted by taking the expectation value of $H_{m q}$ in Eq. (9), but it is more instructive and simpler to stand by our basic assumption that
$2 q_{20}$ is an acceptable quantum number and rely on the exact SU3 result as a guide,

$E=-\frac{\hbar \omega \kappa}{\mathcal{N}_{2 q}^{2}}[2 \lambda(2 \lambda+6)+2 \mu(2 \mu+6)+4 \lambda \mu-3 L(L+1)]$,

where $\lambda$ and $\mu$ are the difference in quanta in the $z$ and $x$ and the $x$ and $y$ directions, respectively. This result is valid for the $q \cdot q$ force that contains one- and two-body parts. Because we are not interested in the former, we expect modifications if they are neglected. Moreover, we restrict the energy estimates to $(\lambda 0)$ representations because the only obviously correct identification in the absence of external monopole fields is $2 \lambda=\left\langle 2 q^{20}\right\rangle$. The idea is to assume that the quadrupole contribution to the energy keeps this form using the calculated $\left\langle 2 q^{20}\right\rangle$ value.

The proposed estimates are as follows:

$$
\begin{aligned}
B E 2 & =B\left(E 2: 2^{+} \rightarrow 0^{+}\right)=\left[\left(\left\langle 2 q_{20}\right\rangle+3\right) b^{2}\right]^{2} / 50.3, \\
Q_{s} & =Q_{(J=2) s}=\left(\left\langle 2 q_{20}\right\rangle+3\right) b^{2} / 3.5, \\
E & =\left\langle H_{\mathrm{sp}}\right\rangle-\frac{\hbar \omega \kappa}{\mathcal{N}_{2 q}^{2}}\left\langle 2 q_{20}\right\rangle\left(\left\langle 2 q_{20}\right\rangle+\zeta\right) .
\end{aligned}
$$

For $Q_{s}$ and $B\left(E 2: 2^{+} \rightarrow 0^{+}\right)$, we use Eqs. (7) and (8). The norms are those of the full quadrupole interaction; i.e., $\mathcal{N}_{2 q}=$ $\sqrt{2.5} \mathcal{N}_{2 q 20}$. The parameter $\zeta$ in the form of $E$ should be 6 if the SU3 analogy held perfectly. However, as hinted above and made evident in Sec. IV C, this is not possible and $\zeta$ must be viewed as an artifact to estimate uncertainties in the guessed energies.

Finally, let us propose a generalization of Eq. (16) for the the energy of a $r_{3}^{k}(g d s)^{l}$ configuration and write explicitly $Q_{s}$ and $B\left(E 2: 2^{+} \rightarrow 0^{+}\right)$. Calling $\left\langle 2 q_{20(i)}\right\rangle=Q_{i}$, we have

$$
\begin{aligned}
B E 2 & =B\left(E 2: 2^{+} \rightarrow 0^{+}\right)=\left[\left(Q_{3}+Q_{4}+3\right) b^{2}\right]^{2} / 50.3, \\
Q_{s} & =Q_{(J=2) s}=\left(Q_{3}+Q_{4}+3\right) b^{2} / 3.5, \\
E & =H_{m}-\hbar \omega \kappa\left(\frac{Q_{3}}{15}+\frac{Q_{4}}{23}\right)\left(\frac{Q_{3}+\zeta}{15}+\frac{Q_{4}+\zeta}{23}\right),
\end{aligned}
$$

where the monopole term $H_{m}$ subsumes the evolving behavior of the single-particle fields discussed in Sec. V B.

\section{Pseudo- and quasi-SU3 as exact symmetries}

According to $\mathrm{SU} 3,{ }^{28} \mathrm{Si}$ has a prolate-oblate degenerate ground state corresponding to the $(\lambda, \mu)=(12,0)$ and $(0,12)$ representations. This holds for the full $q \cdot q$, i.e., including both its one- and two-body terms. If the former are ignored we obtain the results in the top right part of Table III, which show no signs of an exact degeneracy. The estimated energy using $\zeta=0$ in Eq. (16) is about 5\% larger than the exact one. Nearly perfect degeneracy is achieved with the monopole-free $q \cdot q$-i.e., with all centroid averages set to 0 -in the top left part and the estimated energy with $\zeta=3$ is now some $5 \%$ too small.

The story repeats itself in the bottom parts for ${ }^{68} \mathrm{Se}$ : a remarkable result establishing that pseudo-SU3 behaves as an exact symmetry in this case. This is a puzzling result because 
TABLE III. $q \cdot q$ calculations in ${ }^{28} \mathrm{Si}\left[(s d)^{12}\right]$ (top parts) and ${ }^{68} \mathrm{Se}\left[r_{3}^{12}\right]$ (bottom parts). To the left, $q \cdot q$ is made monopole free. "Int" stands for intrinsic values defined in Eq. (16), with $\zeta=3$ in the left part and 0 in the right one. Absolute energies given for the ground state; excitation energies given for the other states $(\mathrm{MeV})$.

\begin{tabular}{|c|c|c|c|c|c|c|c|}
\hline$J$ & $E$ & $Q_{s}$ & $B E 2$ & $J$ & E & $Q_{s}$ & $B E 2$ \\
\hline 0 & -27.26329 & & & 0 & -22.044 & & \\
\hline 0 & 0.00192 & & & 2 & 0.958 & -26.330 & 166.979 \\
\hline 2 & 0.91714 & -0.7785 & 167.299 & 0 & 1.646 & & \\
\hline 2 & 0.91730 & 0.7785 & 167.301 & 2 & 2.494 & 26.323 & 166.903 \\
\hline Int & -26.20 & -26.40 & 169.74 & Int & -23.23 & -26.40 & 169.74 \\
\hline 0 & -14.97822 & & & 0 & -12.176 & & \\
\hline 0 & 0.00042 & & & 2 & 0.533 & -42.087 & 426.802 \\
\hline 2 & 0.50677 & -2.8707 & 427.591 & 0 & 0.996 & & \\
\hline 2 & 0.50683 & 2.8708 & 427.590 & 2 & 1.467 & 42.117 & 426.915 \\
\hline Int & -13.99 & 41.21 & 413.64 & Int & -12.73 & 41.21 & 413.64 \\
\hline
\end{tabular}

we are using the true $q \cdot q$ potential whose matrix elements coincide in magnitude with their pseudo counterparts but have different sign structure, so much so that their overlaps [in the sense of Ref. [11], Eq. (44)] nearly vanish.

Let us draw some conclusions.

(i) Energies are very sensitive to monopole behavior, but $B\left(E 2: 2^{+} \rightarrow 0^{+}\right)$rates are not.

(ii) When bands-with equal $B\left(E 2: 2^{+} \rightarrow 0^{+}\right)$and opposite $Q_{s}$-cross, they mix, leading to unchanged $B\left(E 2: 2^{+} \rightarrow 0^{+}\right)$and cancellation of $Q_{s}$. Note that this could happen through small "impurities" in the Hamiltonian. If the symmetry were exact, the Lanczos algorithm used in the diagonalizations could not break the degeneracy.

(iii) Pseudo-SU3 appears to be close to an exact symmetry.

\section{Checks}

Allowing the single-particle energies to vary produces more stringent tests of the estimates in Eq. (16). Numerous calculations done for the $r_{3}^{n}$ and $(g d s)^{n}$ spaces lead to results that are well summarized by the examples in Table IV.

TABLE IV. Monopole-free $q \cdot q$ calculations in $(g d s)^{8-12}$. Single-particle energies in $\mathrm{MeV}: \varepsilon_{i}=[0.0,0.0$, and 0.0] (e0) and [0.0, 1.0, and 2.0] (e1) for $i=g, d, s$, respectively. "Int" stands for intrinsic values defined in Eq. (16), with $\zeta=3$.

\begin{tabular}{|c|c|c|c|c|c|c|c|}
\hline$J$ & $E$ & $\begin{array}{c}Q_{s} \\
g d s)^{8} \mathrm{e} 0\end{array}$ & $B E 2$ & $J$ & $E$ & $\begin{array}{c}Q_{s} \\
g d s)^{8} \mathrm{e} 1\end{array}$ & $B E 2$ \\
\hline 0 & -12.977 & & & 0 & -8.976 & & \\
\hline 2 & 0.113 & -59.735 & 857.959 & 2 & 0.103 & -57.313 & 795.566 \\
\hline Int & -12.34 & $\begin{array}{r}-59.98 \\
g d s)^{12} \mathrm{e} 0\end{array}$ & 876.05 & Int & -8.28 & $\begin{array}{r}-57.50 \\
g d s)^{12} \mathrm{e} 1\end{array}$ & 805.07 \\
\hline 0 & -17.894 & & & 0 & -12.641 & & \\
\hline 2 & 0.125 & -65.296 & 1161.574 & 2 & 0.136 & -65.609 & 1065.721 \\
\hline Int & -15.84 & -69.16 & 1165.04 & Int & -8.63 & -67.49 & 1109.34 \\
\hline
\end{tabular}

In all cases the self-consistent calculations do well for the quadrupole properties. The estimated energies in $(g d s)^{8}$ are also satisfactory, but they fall short for $(g d s)^{12}$, which is triaxial because the platform at $\left\langle 2 q^{20}\right\rangle=1.5$ in Fig. 3 is not fully filled, leading to $\mu \neq 0$, while $E$ in Eq. (16) it is designed for $\mu=0$ only.

\section{V. $N=Z$ NUCLEI}

Granted the benefit of some hindsight, a reading of Fig. 3 suggests three regimes for $N=Z$ nuclei from ${ }^{56} \mathrm{Ni}$ up to ${ }^{96} \mathrm{Cd}$. Note that placing the $g d s$ "quasi" orbits on top of the "pseudo" $r_{3}$ ones was designed to facilitate such a reading.

(i) The $r_{3}$ pseudo-SU3 nuclei. They fill orderly the three lowest levels in Fig. 3: ${ }^{60} \mathrm{Zn}$ (analog of ${ }^{20} \mathrm{Ne}$ in the $s d$ shell, a mild rotor), ${ }^{64} \mathrm{Ge}$ (analog of ${ }^{24} \mathrm{Mg}$, a rotor exhibiting a $\gamma$ band, as expected whenever orbits are not all filled at a given level), ${ }^{68} \mathrm{Se}$ (analog of ${ }^{28} \mathrm{Si}$, with degenerate prolate and oblate bands). While SU3 dominance is largely frustrated in the $s d$ shell, here it is expected to hold well because of the near degeneracy of the single-particle orbits. This region makes it possible to study the full $p f$ to $r_{3}$ reduction, a unique opportunity to validate the notion of model space and, in particular, the assumption that ${ }^{56} \mathrm{Ni}$ can be treated as a closed shell. As for $r_{3} g$ calculations [22], they add little to the $r_{3}$ ones.

(ii) Coexistence from ${ }^{72} \mathrm{Kr}$ to ${ }^{84} \mathrm{Mo}$. For 12 particles, i.e., ${ }^{68} \mathrm{Se},\left\langle 2 q^{20}\right\rangle$ reaches a maximum in $r_{3}$ (see the last lines of Tables I and II). Adding particles to the pseudo orbits leads to a loss while adding them to the quasi orbits leads to a gain. By filling the quasi orbits, well-deformed prolate states can be constructed for $4,8,12$, and 16 particles whose quadrupole energy will overcompensate the monopole (i.e., single-particle) losses. Oblate states very close in energy can also be found, leading to coexisting bands. The prolate and oblate states demand $r_{3} g d s$ and $r_{3} g$ spaces, respectively. The associated dimensionalities exceed $10^{14}$ for the former and $10^{10}$ for the latter, still large but feasible. Therefore, we rely on a generalization of the simplified approach of Sec. IV C for both deformations and check the oblate results via exact diagonalizations. For studies of the region, see Refs. [23,24].

(iii) The $r_{3}$ g nuclei ${ }^{88} \mathrm{Ru},{ }^{92} \mathrm{Pd}$, and ${ }^{96} \mathrm{Cd}$. The second has been measured recently [25] and postulated as candidate for a new form of boson aligned collectivity. We examine the claim. The-still unknown-spectrum of ${ }^{96} \mathrm{Cd}$ is shown to be probably closer to $s d g$ than to $r_{3} g$.

\section{A. The $p f$-to- $r_{3}$ reduction}

Doubts may be raised about the doubly magic nature of ${ }^{56} \mathrm{Ni}$ because its first $2^{+}$is rather low and, depending on the effective interaction used (KB3G, GXPF1A) [26,27], the closed-shell component amounts to only $60 \%-70 \%$. However, it is in the nature of the shell model to recognize that there may 
be a difference between the potentially complicated structure of a state and its simple behavior. As a first hint of what is expected of magic nuclei, we refer to Figs. 1-5 in Ref. [28]: At magic numbers, two-neutron and two-proton separation energies exhibit systematic jumps, clearly the case for $N$ or $Z=28$ and a fortiori for ${ }^{56} \mathrm{Ni}$, but not for occasional candidates such as $N=56$, which is magic only for $Z=40$.

For our present purpose the state of interest is the head of the $4 \mathrm{p}-4 \mathrm{~h}$ rotational band. According to Eq. (6), four holes in the $0 f_{7 / 2}$ orbit give a prolate contribution of $12 b^{2}$ to the intrinsic quadrupole moment while four pseudo-SU3 particles in $r_{3}$ contribute with $\approx 22 b^{2}$, adding up to $34 b^{2}$, in agreement with $32 b^{2}$ from a full $4 \mathrm{p}-4 \mathrm{~h} p f$-shell calculation, a first example of the use of our schematic coupling schemes.

\section{1. $0 \hbar \omega$ polarization}

The most important characteristic of a doubly magic nucleus is that it defines a before and an after. Before ${ }^{56} \mathrm{Ni}$, nuclei are basically of $f$ type. Beyond, they are at first of $r_{3}$ type until the extension to $r_{3} g d s$ spaces becomes imperative. To treat ${ }^{56} \mathrm{Ni}$ as a core, the Hamiltonian and transition operators have to be renormalized. The dominant mechanism involves coupling to the low-lying $2^{+}$state, leading to three-body forces and two-body effective transition operators [29] (i.e., state-dependent effective charges), whose neglect, as stressed in Ref. [14], is “common but bad practice.” Short of a rigorous treatment, we chose the following expediencies.

(i) For the energies we assume that JUN45 [17] provides a reasonable approximation to the effective Hamiltonian. To fix ideas: In Ref. [14] it is shown that for the quadrupole component of the bare realistic forces the $2 \hbar \omega$ effects demand a $30 \%$ boost (consistent with what is known about phenomenological interactions). As a consequence, the effective $q \cdot q$ amounts to about $50 \%$ of the total interaction. In the case of JUN45 it jumps to over $75 \%$, indicating a strong contribution owing to $0 \hbar \omega$ mechanisms.

(ii) For the transition operators we proceed by brute force, estimating effective charges by comparing full $p f$ transitions rates to those obtained in the $r_{3}$ or $r_{3} g$ spaces.

\section{2. ${ }^{60} \mathrm{Zn}$, more on magicity}

To check that ${ }^{60} \mathrm{Zn}$ is properly described by $r_{3}^{4}$ configurations we do a full $p f$ diagonalizations which involves $2292604744 M=0$ Slater determinants. The story is told in Table V. A calculation in the $r_{3}$ space, using a pure quadrupolequadrupole interaction, gives values in the range $24 b^{2}$.

TABLE V. Properties of the yrast band of ${ }^{60} \mathrm{Zn}(E$ in $\mathrm{MeV}, Q$ in units of $b^{2}$ ). Calculations: full $p f$ with $\mathrm{KB} 3 \mathrm{GR}$; and $r_{3}$ with $q \cdot q$.

\begin{tabular}{cccccccc}
\hline \hline $\mathrm{J}$ & $E_{\exp }$ & $E_{q q}$ & $E_{p f}$ & $Q_{0 s, q q}$ & $Q_{0 s, p f}$ & $Q_{0 t, q q}$ & $Q_{0 t, p f}$ \\
\hline $2^{+}$ & 1.00 & 1.00 & 1.07 & 24 & 22 & 23 & 31 \\
$4^{+}$ & 2.19 & 3.34 & 2.31 & 23 & 25 & 22 & 30 \\
$6^{+}$ & 3.81 & 7.03 & 4.06 & 23 & 14 & 19 & 31 \\
\hline \hline
\end{tabular}

As expected, we have good rotational features including $J(J+1)$ spacings. The full $p f$-shell calculation using the KB3GR interaction [30] accounts well for the experimental spectrum. The $J(J+1)$ spacings are gone, but this is of little consequence. As abundantly emphasized in Ref. [3], what matters is the wave function, i.e., the quadrupole moments. The spectrum may be sensitive to details detected in first-order perturbation theory that do not change the structure of the state. The message from Table $\mathrm{V}$ is that the quadrupole moments of the huge calculation and the modest one are compatible, to within a crucial caveat: The full $p f$ space leads to $Q_{0 t}$ values that are about 1.36 times bigger than the $r_{3}$ ones. Because the coupling is mediated basically by the $p_{3 / 2} f_{7 / 2}^{-1}$ jumps, the renormalization decreases as the $p_{3 / 2}$ orbit gets filled, thus blocking the jumps. The results hardly change when JUN45 is used instead of $q \cdot q$ in the $Q_{0 t, q q}$ column of Table V: 23 goes to 20.8 , increasing the enhancement factor $F$ from 1.36 to 1.48. The calculated spectrum-though still dilated-comes closer to the experimental one.

Note that the evolutions of $Q_{0 s}$ and $Q_{0 t}$ are quite different. In general, the two quantities will be approximately equal only in the case of well-developed rotors. More often than not, $Q_{s}$ is very sensitive to details, while $Q_{t}$ is close to the predictions from Tables I and II.

It is worth mentioning that ${ }^{60} \mathrm{Zn}$ has a superdeformed excited band at relatively low energy with $Q_{0}=67(6) b^{2}$ [31]. From Tables II and III two prolate candidates emerge with configurations $f^{12} r_{3}^{4}(g d s)^{4}$ and $f^{12}(g d s)^{8}$. Both are consistent with observation.

\section{3. ${ }^{64} \mathrm{Ge}$}

For ${ }^{64} \mathrm{Ge}$ the diagonalization of the $q \cdot q$ interaction in the $\left(r_{3}\right)^{8}$ space yields the expected results for an (84) SU3 representation with nearly degenerate $2^{+}$states-with $Q_{0}$ of equal magnitude and opposite signs - corresponding to the $K=0$ and 2 ground-state and $\gamma$ bands, respectively, and $B\left(E 2: 2^{+} \rightarrow 0^{+}\right)$of about $300 e^{2} \mathrm{fm}^{4}$. Table VI proposes a comparison of $q \cdot q$ and JUN45 results - in $r_{3}$ and $r_{3} g$ spaces, respectively - with data, well reproduced by GXPF1A calculations [32]. Using as reference the $B\left(E 2: 2^{+} \rightarrow 0^{+}\right)$values, it is found that in going from $r_{3}$ to $p f$ the enhancement factors $F$ are 1.62 (for JUN45) and 1.23 (for $q \cdot q$ ).

TABLE VI. Properties of low-lying states in ${ }^{64} \mathrm{Ge}$ : energies in $\mathrm{MeV}, B(E 2)$ in $e^{2} \mathrm{fm}^{4}$. Calculations: full $p f$ with GXPF1A [32]; $r_{3} g$ with JUN45; and $r_{3}$ with $q \cdot q$.

\begin{tabular}{cccccc}
\hline \hline$J^{\pi}$ & & $\operatorname{Exp}$ & $p f$ & $r_{3} g$ & $q \cdot q$ \\
\hline $2_{1}^{+}$ & $E_{x}$ & 0.90 & 0.94 & 0.86 & 0.50 \\
$2_{1}^{+}$ & $Q_{s}$ & & -18.6 & -24.4 & 5.03 \\
& $B\left(E 2: 2_{1}^{+} \rightarrow 0_{1}^{+}\right)$ & $410(60)$ & 406 & 251 & 300 \\
$2_{2}^{+}$ & $E_{x}$ & 1.579 & 1.56 & 1.27 & 0.55 \\
$2_{2}^{+}$ & $Q_{s}$ & & 18.5 & 23.3 & -5.42 \\
& $B\left(E 2: 2_{2}^{+} \rightarrow 2_{1}^{+}\right)$ & $620(210)$ & 610 & 182 & 479 \\
& $B\left(E 2: 2_{2}^{+} \rightarrow 0_{1}^{+}\right)$ & $1.5(5)$ & 14 & 13 & 39 \\
$4_{1}^{+}$ & $E_{x}$ & 2.053 & 2.00 & 2.16 & 1.61 \\
& $B\left(E 2: 4_{1}^{+} \rightarrow 2_{1}^{+}\right)$ & & 674 & 314 & 390 \\
\hline \hline
\end{tabular}


TABLE VII. Properties of low-lying states in ${ }^{68}$ Se: energies in $\mathrm{MeV}, B(E 2)$ in $e^{2} \mathrm{fm}^{4}$. Calculations: full $p f$ with GXPF1A [32]; $r_{3}$ with JUN45: $r_{3}$ with $q \cdot q$; and full $p f$ with KB3GR $(P F)$. The experimental $0_{2}^{+}$energy is a guess.

\begin{tabular}{ccccccc}
\hline \hline$J^{\pi}$ & & Exp & $p f$ & $r_{3}$ & $q \cdot q$ & $P F$ \\
\hline $0_{2}^{+}$ & $E_{x}$ & $(1.19)$ & 0.69 & 0.96 & 0.79 & 1.42 \\
$2_{1}^{+}$ & $E_{x}$ & 0.85 & 0.71 & 0.54 & 0.53 & 0.96 \\
$2_{1}^{+}$ & $Q_{s}$ & & 11 & 35 & -42 & 39 \\
& $B\left(E 2: 2_{1}^{+} \rightarrow 0_{1}^{+}\right)$ & $440(60)$ & 491 & 307 & 420 & 409 \\
$2_{2}^{+}$ & $E_{x}$ & 1.59 & 1.00 & 1.39 & 1.26 & 1.74 \\
$2_{2}^{+}$ & $Q_{s}$ & & -8 & -33 & 42 & -16 \\
& $B\left(E 2: 2_{2}^{+} \rightarrow 2_{1}^{+}\right)$ & & 689 & 7 & 0.00 & 297 \\
& $B\left(E 2: 2_{2}^{+} \rightarrow 0_{2}^{+}\right)$ & & 499 & 262 & 420 & 223 \\
& $B\left(E 2: 2_{2}^{+} \rightarrow 0_{1}^{+}\right)$ & 0.3 & 4 & 0.7 & 0.00 & 10 \\
$4_{1}^{+}$ & $E_{x}$ & 1.94 & 1.66 & 1.61 & 1.77 & 1.86 \\
$4_{1}^{+}$ & $Q_{s}$ & & 59 & 43 & -53 & 63 \\
& $B\left(E 2: 4_{1}^{+} \rightarrow 2_{1}^{+}\right)$ & & 590 & 419 & 565 & 810 \\
$4_{2}^{+}$ & $E_{x}$ & 2.55 & 1.98 & 2.28 & 2.37 & 2.79 \\
$4_{2}^{+}$ & $Q_{s}$ & & -51 & -42 & 53 & -14 \\
& $B\left(E 2: 4_{2}^{+} \rightarrow 2_{2}^{+}\right)$ & & 510 & 354 & 565 & 154 \\
\hline \hline
\end{tabular}

\section{4. ${ }^{68}$ Se: The double platform}

The structure of $N=Z$ even nuclei from $A=72$ to 84 is described by piling up $(g d s)^{4}$ blocks on top $r_{3}^{12}$, i.e., on top of either the oblate and prolate ground-state bandscorresponding to the $(12,0)$ and $(0,12)$ SU3 representationsof ${ }^{68} \mathrm{Se}$, which becomes a common "double platform" (refer to Fig. 3). Hence the importance of this nucleus to fix the $e_{0}$ effective charge.

From Table I the estimate $Q_{0} / b^{2}= \pm 33.2$, i.e., $B(E 2$ : $\left.2^{+} \rightarrow 0^{+}\right) \approx 414 e^{2} \mathrm{fm}^{4}$, consistent the $q \cdot q$ numbers in Table VII, which also collects JUN45 results in $r_{3}$, the full $p f$ GXPF1A and KB3GR ones (labeled $p f$ and $P F$, respectively) and data including the only experimentally known $B(E 2$ : $\left.2^{+} \rightarrow 0^{+}\right)=440(60) e^{2} \mathrm{fm}^{4}$.

With the exception of the $B\left(E 2: 2_{2}^{+} \rightarrow 2_{1}^{+}\right)$the calculations in $r_{3}$ and $p f$ are quite consistent, with enhancement factors $F \approx 1.16$ and 1.38 for the $q \cdot q$ and JUN45 numbers, respectively. The KB3GR interaction yields somewhat better spectra than GXPF1A, and similar quadrupole properties except for the $J=2_{2}^{+}$and $4_{2}^{+}$states that are more mixed for the latter.

Using the $2 \hbar \omega$ value $e_{0}=1.77$ [14], the $0 \hbar \omega$ contribution increases it to $e_{0}=1.77 \sqrt{F} \approx 2.1 \pm 0.1$. When $g d s$ particles come into play their quadrupole operators will also couple with the $J=2^{+}$state in ${ }^{56} \mathrm{Ni}$, though more weakly owing to larger norm denominators [see Eqs. (9) and (17)]. It is hoped that the associated suppression can be accommodated by the proposed estimate.

The JUN45 calculation in $r 3 g$ leads to a ground state that is $60 \% 0 \mathrm{p}-0 \mathrm{~h}, 30 \%$ 2p-2h, and $10 \%$ 4p-4h. As can be gathered from Tables I and II, these admixtures bring no extra oblate coherence, but with the same numbers prolate contributions could make a difference in a full $r_{3} g d s$ calculation. VAMPIR calculations [33] indicate substantial oblate-prolate mixing in the ground-state band. Further data on this nucleus could be of interest.
TABLE VIII. Properties of $r_{3}^{k}(g d s)^{l}$ configurations. Total $(E)$, quadrupole $\left(E_{q}\right)$, and single-particle $(h s p)$ energies from Eq. (18) with $\zeta=0$, in $\mathrm{MeV}$; quadrupole moment $Q_{4}=\left\langle 2 q_{20(4)}\right\rangle ; B E 2=$ $B\left(E 2: 2^{+} \rightarrow 0^{+}\right)$in $e^{2} \mathrm{fm}^{4}$ from Eq. (17); $\beta=8$ in Eq. (14). For prolate states $Q_{4}$ is the calculated one. For oblate states the space is $r_{3}^{k}(g)^{l}$, so $\epsilon_{r_{3}}=0.0$ and $Q_{4}$ (not shown) is from Table II. $Q_{3}$ is always from Table II. Energies of triaxial states are in boldface. Single-particle energies in MeV: $\varepsilon_{i}=0.0,3.0$, and 4.0 [0.0, 4.0, and 5.0] for $i=g, d, s$, respectively, and $\epsilon_{g r}=2.5$ [2.0]. Numbers in square brackets apply to the last two lines only.

\begin{tabular}{ccccccccc}
\hline \hline$k$ & $l$ & $A$ & $E$ & $-E_{q}$ & $h s p$ & $Q_{3}$ & $Q_{4}$ & $B E 2$ \\
\hline 12 & 4 & 72 & -12.29 & 25.53 & 3.24 & 30.20 & 23.00 & 1225 \\
16 & 0 & 72 & -8.37 & 8.37 & 0.0 & & & 324 \\
12 & 4 & 72 & -10.63 & 20.63 & 0.0 & & & 939 \\
12 & 8 & 76 & -12.29 & 40.05 & 7.76 & 30.20 & 41.17 & 2212 \\
16 & 4 & 76 & -2.46 & 15.62 & 3.15 & 20.72 & 22.85 & 867 \\
14 & 6 & 76 & -4.90 & 19.90 & 0.0 & & & 987 \\
16 & 4 & 76 & -6.23 & 16.23 & 0.0 & & & 805 \\
12 & 12 & 80 & $-\mathbf{0 . 3 0}$ & 47.01 & 16.71 & 30.20 & 49.15 & 2792 \\
16 & 8 & 80 & 1.76 & 27.45 & 7.69 & 20.72 & 41.17 & 1733 \\
18 & 6 & 80 & -0.04 & 15.04 & 0.0 & & & 823 \\
12 & 16 & 84 & 5.91 & 51.92 & 17.82 & 30.20 & 54.71 & 3271 \\
16 & 12 & 84 & $\mathbf{1 3 . 4 7}$ & 33.20 & 16.67 & 20.72 & 49.01 & 2240 \\
20 & 8 & 84 & 6.05 & 13.95 & 0.0 & & & 840 \\
12 & 16 & 84 & 3.02 & 51.25 & 22.26 & 30.20 & 54.05 & 3223 \\
20 & 8 & 84 & 2.05 & 13.95 & 0.0 & & & 840 \\
\hline \hline
\end{tabular}

\section{B. The central region: $A=72$ to 84}

Let us recast $E$ in Eq. (17) so as to separate the two basic contributions to the monopole term $H_{m}$,

$$
\begin{gathered}
E=\sum \varepsilon_{i=g, d, s}\left\langle n_{i}\right\rangle+l\left(\varepsilon_{g}-\varepsilon_{r_{3}}\right) \\
-\hbar \omega \kappa\left(\frac{Q_{3}}{15}+\frac{Q_{4}}{23}\right)\left(\frac{Q_{3}+\zeta}{15}+\frac{Q_{4}+\zeta}{23}\right) \\
=h s p+l \epsilon_{g r}+E_{q},
\end{gathered}
$$

where we have introduced the notations used in Table VIIIthe core of this study - which lists the properties of the dominant and subdominant prolate and oblate states.

To ascertain the stability of the estimates, all the calculations, done with $\zeta=0$, have been redone for $\zeta=3$. The examples that follow are for $A=84$, which involves the largest magnitudes for $h s p, l \epsilon_{g r}$, and $E_{q}$ and hence, presumably, the largest uncertainties.

For $\zeta=0 \rightarrow 3$ the energies $E=5.91,13.47$, and 6.05 in Table VIII go to $4.03,11.89$, and 5.0, respectively, leaving unchanged the qualitative conclusions that may be drawn.

The evolution of the monopole is another source of uncertainty: The $\varepsilon_{i}$ and $\varepsilon_{g r}$ numbers are suggested by GEMO [34] at the beginning of the region. As the $g$ filling increases, the orbit will separate from its $d s$ partners and come closer to the $r_{3}$ space. To simulate this effect, in the last two lines of Table VIII the single-particle energies are changed to the bracketed values in the caption. As a consequence the energies at $E=5.91$ and 6.05 change to 3.02 and 2.05, respectively. Again the qualitative conclusions are not affected. 
These results for ${ }^{84} \mathrm{Mo}$ are typical and illustrate two important points.

(1) For prolate states $B\left(E 2: 2^{+} \rightarrow 0^{+}\right)$and $\left\langle 2 q^{20}\right\rangle$ are very unsensitive to monopole behavior and hence remain close to their theoretical quasi + pseudo-SU3 maxima. In our example $Q_{4}=54.71$ and 54.05 against the 55.88 maximum.

(2) Energies of prolate states are very sensitive to the single-particle field $H_{\text {sp }}$. In our example there is a shift of some $4.5 \mathrm{MeV}$ : $h s p=17.82$ vs $22.26 \mathrm{MeV}$. However, the relative positions of the states remain fairly stable.

Examine now what conclusions can be drawn from Table VIII.

${ }^{72} \mathrm{Kr}$. This is the only species where $B\left(E 2: 2^{+} \rightarrow 0^{+}\right)$are close for prolate and oblate candidates. Probable coexistence.

${ }^{76} \mathrm{Sr}$. There is a single candidate. It is an experimentally superb rotor with good $J(J+1)$ sequence, in perfect agreement of Table VIII with a recent measure: $B\left(E 2,2^{+} \rightarrow 0^{+}\right)=$ 2220(270) $e^{2} \mathrm{fm}^{4}$ [35].

${ }^{80} \mathrm{Zr}$. The lowest state is expected to gain some $4 \mathrm{MeV}$ because of triaxiality and the observed rotational spectrum seems to guarantee $B\left(E 2: 2^{+} \rightarrow 0^{+}\right)$, close to the prediction. However, the very low-lying oblate state may blur the picture. Moreover, the prolate $8 \mathrm{p}-8 \mathrm{~h}$ (and a $10 \mathrm{p}-10 \mathrm{~h}$, not shown) are also close and triaxial. Finally, the frustrated doubly magic $N=Z=40$ is at $0 \mathrm{MeV}$. This is a very interesting nucleus.

${ }^{84} \mathrm{Mo}$. There is a strong hint of coexistence, even triple coexistence through gains owing to triaxiality of the second prolate candidate.

Except for ${ }^{76} \mathrm{Sr}$, coexistence is expected in the other nuclei and is examined in Sec. VI.

\section{The $r 3 g$ calculations}

Calculations in the $\left(r_{3} g\right)^{n}$ spaces have been carried out for all $n$. We concentrate on results for $A \geqslant 80$. In particular, ${ }^{80} \mathrm{Zr}$ and ${ }^{84} \mathrm{Mo}$ are mainly of interest in lending support to a basic observation about oblate bands.

Contrary to prolate states that privilege maximizing the deformation, the oblate bands give precedence to mixing that reduces it. As a consequence, our schematic estimates overestimate $\left\langle 2 q^{20}\right\rangle$ and $B\left(E 2: 2^{+} \rightarrow 0^{+}\right)$and underestimate energies.

\section{1. ${ }^{80} \mathrm{Zr}$ and ${ }^{84} \mathrm{Mo}$}

In Table IX the extracted $Q_{0} \approx 180$ is definitely lower than the $6 \mathrm{p}-6 \mathrm{~h}$ number from Table II, $Q_{0} \approx(19+23+3) b^{2} \approx 203$ $e \mathrm{fm}^{2}$. The wave functions have $22 \% 4 \mathrm{p}-4 \mathrm{~h}, 44 \% 6 \mathrm{p}-6 \mathrm{~h}$, and

TABLE IX. ${ }^{80} \mathrm{Zr}$. Results of the full $\left(1.1 \times 10^{10}\right.$-dimensional $) r_{3} g$ calculation with JUN45 ( $E$ 's in $\mathrm{MeV}, Q$ in $e \mathrm{fm}^{2}$ and $B\left(E 2: 2^{+} \rightarrow\right.$ $\left.0^{+}\right)$in $e^{2} \mathrm{fm}^{4}$ ).

\begin{tabular}{cccccc}
\hline \hline$J$ & $E\left(2^{+}\right)$ & $Q_{s}$ & $Q_{0 s}$ & $B(E 2)$ & $Q_{0 t}^{2}$ \\
\hline 2 & 0.393 & 51 & -179 & 642 & $(180)^{2}$ \\
\hline \hline
\end{tabular}

TABLE X. ${ }^{84}$ Mo. Properties of the yrast band; experiment vs calculations in the $r_{3} g$ space with the JUN45 interaction: To the left, truncated up to four holes in $r_{3}$; to the right, complete space [ $E$ in $\mathrm{MeV}$, $Q$ in $e \mathrm{fm}^{2}$, and $B(E 2)$ in $\left.e^{2} \mathrm{fm}^{4}\right] . E_{x}$ and $E_{t}$ stand for experimental and theoretial energies respetively.

\begin{tabular}{llcccccccc}
\hline \hline$J$ & $\mathrm{Ex}$ & $\mathrm{Et}$ & $-Q_{0 s}$ & $B(E 2)$ & $-Q_{0 t}$ & $\mathrm{Et}$ & $-Q_{0 s}$ & $B(E 2)$ & $-Q_{0 t}$ \\
\hline 0 & 0.0 & 0.0 & & & & 0.00 & & & \\
2 & 0.44 & 0.17 & 194 & 762 & 196 & 0.29 & 189 & 708 & 188 \\
4 & 1.12 & 0.56 & 190 & 1081 & 195 & 0.84 & 189 & 1020 & 189 \\
6 & 2.01 & 1.15 & 184 & 1179 & 194 & 1.60 & 189 & 1118 & 189 \\
\hline \hline
\end{tabular}

$28 \% 8 \mathrm{p}-8 \mathrm{~h}$. Mixing with prolate states nearby may be at the origin of the reduction, as confirmed in Table $\mathrm{X}$ for ${ }^{84} \mathrm{Mo}$.

The ground-state band is dominated now by the $r_{3}^{-4} g^{8}$ configuration. From Table II, $Q_{0} \approx(21+20+3) b^{2}=44 \times$ $4.61 \approx 203 e \mathrm{fm}^{2}$, not inconsistent with the truncated calculations (left part of the table) that exhibit good rotational features. Once the full space is incorporated (right part of the table), the energies depart from the $J(J+1)$ sequence, while the quadrupole properties, still those of a rotor, have suffered an erosion owing to the inclusion of prolate states as suggested in ${ }^{80} \mathrm{Zr}$.

\section{2. ${ }^{88} R u$}

In ${ }^{88} \mathrm{Ru}$ we come at last to a genuine $r_{3} g$ nucleus. (Note that for $A \geqslant 88$ most numerical results reported below duplicate those of the Rutgers group [36].) Table XI corresponds to an yrast oblate band exhibiting $50 \% r_{3}^{-4} g^{12}$ oblate dominance. This is not obvious, because $g^{12}$ is now beyond midshell and the largest $\left\langle 2 q^{20}\right\rangle$ is prolate. However, the oblate $\left\langle 2 q^{20}\right\rangle$ in $r_{3}^{-4}$ is sufficiently strong to dominate but the prolate admixtures distort and reduce the original $\left\langle 2 q^{20}\right\rangle=-(18.66+20.24)$ and $Q_{0} \approx-182$ to $Q_{0 s} \approx-125$ and $Q_{0 t} \approx-160$ in Table XI. It is seen that in this nucleus the prolate-oblate competition within the $r_{3} g$ space is played up. ${ }^{92} \mathrm{Pd}$ will bring further news.

\section{3. ${ }^{92} P d$}

The authors' interest in heavy $N=Z$ nuclei was sparked by the first measurement of the ${ }^{92} \mathrm{Pd}$ spectrum, accompanied by an interpretation that associated it with a condensate of $\left(g_{9 / 2}^{2}\right)$ neutron-proton pairs coupled to maximum $J=9[25,37,38]$. This raised two issues: that of possible coupling schemes in a $g^{12}$ space and that of possible dominance of this configuration.

TABLE XI. Properties of the yrast band of ${ }^{88} \mathrm{Ru}$ : experiment vs calculations in the $r_{3} g$ space with the JUN45 interaction $[E$ in $\mathrm{MeV}$, $Q$ in $\mathrm{efm}^{2}$, and $B(E 2)$ in $\left.e^{2} \mathrm{fm}^{4}\right]$.

\begin{tabular}{llllc}
\hline \hline$J$ & $E(\exp )$ & $E($ th $)$ & $Q_{s}$ & $B(E 2 \downarrow)($ th $)$ \\
\hline $0_{1}^{+}$ & 0.0 & 0.0 & & \\
$2_{1}^{+}$ & 0.62 & 0.56 & 37 & 492 \\
$4_{1}^{+}$ & 1.42 & 1.31 & 44 & 766 \\
$6_{1}^{+}$ & 2.38 & 2.12 & 47 & 888 \\
$8_{1}^{+}$ & 3.48 & 2.88 & 52 & 980 \\
\hline \hline
\end{tabular}


TABLE XII. Properties of ${ }^{92} \mathrm{Pd}$. Energies in MeV, $Q$ in $e \mathrm{fm}^{2}$ and $B(E 2)$ in $e^{2} \mathrm{fm}^{4}$. Detailed explanation in text.

\begin{tabular}{cccccccccc}
\hline \hline 1 & 2 & 3 & 4 & 5 & 6 & 7 & 8 & 9 & 10 \\
$\mathrm{~J}$ & $\mathrm{Ex}$ & $\mathrm{Et}$ & $\mathrm{Con}$ & $q q$ & $\Omega$ & $B(E 2)$ & $B(E 2)_{r_{3} g}$ & $Q_{s}$ & $Q_{s, r_{3} g}$ \\
\hline 0 & 0.0 & 0.0 & 0.00 & 0.00 & 0.99 & - & - & - & - \\
2 & 0.874 & 0.84 & 0.26 & 0.22 & 0.99 & 225 & 304 & -28 & -3.63 \\
4 & 1.786 & 1.72 & 0.58 & 0.62 & 0.99 & 316 & 382 & -34 & -8.20 \\
6 & 2.563 & 2.52 & 0.85 & 1.20 & 0.98 & 340 & 364 & -31 & -2.77 \\
\hline \hline
\end{tabular}

Table XII — which we comment column by column—sums up sufficient information to resolve both issues:

(1) $J$ value;

(2) experimental spectrum, in very good agreement with 3 ;

(3) JUN45 spectrum;

(4) spectrum of the condensate defined by $-H_{\text {con }}=$ $P_{0}+9 P_{9}$, where $P_{0}$ and $P_{9}$ are the pairing Hamiltonians for $J=0$ and 9 ;

(5) spectrum of the quadrupole force scaled so as to have unit $J=9$ matrix element, close to that of the condensate (within arbitrary scaling factor);

(6) overlap, $\Omega=\langle q q \mid \operatorname{con}\rangle^{2}$, of the wave functions indicating that the condensate and quadrupole coupling schemes are identical (the use of $P_{9}$ should be understood as an artifact to define a coupling scheme; as a Hamiltonian it is better avoided);

$(7,8)$ a Hamiltonian $-H \approx 0.6 q q+0.4 P_{0}$ yields $g^{12}$ energies that are close to the exact ones and $B(E 2)$ that are very close to the pure $q q$ values in column 7 and not too far from the exact ones in column 8, which may encourage the idea of $g^{12}$ dominance in spite of its smallish $30 \%$ contribution to the exact wave function (however, this idea is not supported by the disparity of $Q_{s}$ in columns 9 and 10);

$(9,10)$ spectroscopic $Q_{s}$ for $q q(9)$ and JUN45 [17] (10).

The situation is reminiscent of that of $f_{7 / 2}^{n}$ configurations that yield apparently reasonable energetics and transition rates but quadrupole moments of the wrong sign [39].

The pattern we started following at ${ }^{80} \mathrm{Zr}$ - of oblate states progressively eroded by prolate mixtures-now reaches its climax with the Pyrrhic victory of prolate states practically canceled by oblate mixtures.

\section{4. ${ }^{96} C d$}

For this nucleus, the calculations in the $r_{3} g$ and $g$ spaces with JUN45 give results that are much closer than in ${ }^{92} \mathrm{Pd}$, both for the energies and for the $B(E 2)$ properties and the discrepancies in the spectroscopic quadrupole moments are gone except for the $6^{+}$state.

We have collected some results in Table XIII, adding those from the full $s d g$ space using the Nowacki-Sieja interaction [40], which describes the superallowed decay of ${ }^{100} \mathrm{Sn}$ [41] and the $B(E 2)$ systematics of the light $\mathrm{Sn}$ isotopes [42]. The results for the energies, $B(E 2)$ and $Q$ values vary little between $g$ and $r_{3} g$, pointing to $g$ dominance, not
TABLE XIII. ${ }^{96} \mathrm{Cd}$. Energies in $\mathrm{MeV}, Q$ in $e \mathrm{fm}^{2}$ and $B(E 2)$ in $e^{2} \mathrm{fm}^{4} e^{2} \mathrm{fm}^{4}$.

\begin{tabular}{|c|c|c|c|c|c|c|c|c|c|}
\hline \multirow[b]{2}{*}{$J^{\pi}$} & \multicolumn{3}{|c|}{$\Delta E$} & \multicolumn{3}{|c|}{$B(E 2)$} & \multicolumn{3}{|c|}{$Q_{s}$} \\
\hline & $r_{3} g$ & $g_{9 / 2}$ & $s d g$ & $r_{3} g$ & $g_{9 / 2}$ & $s d g$ & $r_{3} g$ & $g_{9 / 2}$ & $s d g$ \\
\hline $0^{+}$ & 0.0 & 0.0 & 0.0 & & & & & & \\
\hline $2^{+}$ & 0.90 & 0.96 & 0.77 & 152 & 154 & 327 & -19 & -23 & -37 \\
\hline $4^{+}$ & 1.91 & 2.10 & 1.78 & 203 & 206 & 426 & -22 & -22 & -40 \\
\hline $6^{+}$ & 3.02 & 3.08 & 2.78 & 191 & 159 & 351 & -11 & -5 & -23 \\
\hline $8^{+}$ & 3.48 & 3.08 & 3.24 & 47 & 40 & 65 & 40 & 39 & 55 \\
\hline
\end{tabular}

invalidated by the substantial quadrupole coherence brought in by the full $s d g$ space calculation as it amounts basically to an overall scaling.

It is worth mentioning that the latter predicts a $16^{+}$isomer at $5.3 \mathrm{MeV}$.

\section{CASE STUDIES, COMPARISONS, AND PERSPECTIVES}

The central region calls for some extra comments.

\section{A. Coexistence in ${ }^{72} \mathrm{Kr}$}

Exact $\left(r_{3} g\right)^{16}$ calculations with JUN45 [17] for ${ }^{72} \mathrm{Kr}$ indicate that-with respect to Table VIII-the gap $r_{3}^{12} g^{4}-r_{3}^{16}$ is underestimated by about $2 \mathrm{MeV}$, while $B\left(E 2: 2^{+} \rightarrow 0^{+}\right)$is overestimated by $10 \%$. The ground-state band is a nice oblate rotor with nearly constant $Q_{o t} \approx 205 e \mathrm{fm}^{2}, \operatorname{good} J(J+1)$ sequence with the $2^{+}$at $350 \mathrm{keV}$-half the observed valuewhile the $4^{+}$at $1.1 \mathrm{MeV}$ is close to the observed $1.32 \mathrm{MeV}$, while $B\left(E 2: 2^{+} \rightarrow 0^{+}\right)=850 e^{2} \mathrm{fm}^{4}$ against a measured 999(129) $e^{2} \mathrm{fm}^{4}$ [43]. In this reference it is argued that the ground band is oblate. A suggestion that may gain some support from the shape of the Gamow-Teller $\beta^{+}$decay strength function [44]. Recent measures [45] yield $B\left(E 2: 2_{1} \rightarrow 0_{1}\right)=$ $810(150) e^{2} \mathrm{fm}^{4}$ (too small to be prolate) and $B\left(E 2: 4_{1} \rightarrow\right.$ $\left.2_{1}\right)=2720(550) e^{2} \mathrm{fm}^{4}$ (too large to be oblate, even large for prolate in view of theoretical maximum of $2200 e^{2} \mathrm{fm}^{4}$. (Note that an analysis of Fig. 3 of [45] suggests that the 550 $e^{2} \mathrm{fm}^{4}$ error bar is underestimated.)

Clearly, some mixing is necessary and to achieve it we resort to the space which is the largest we could treat and the smallest that could cope with prolate states, i.e., $r_{3}^{16-t}(g d)^{t}$, tractable for $t \leqslant 4$. The interaction chosen is R3GD, i.e., JUN45 supplemented by matrix elements involving the $d$ orbit from the LNPS set [46].

First, two calculations at fixed $t=4$ were made. If the single-particle energy $\varepsilon_{d}$ is set $1.76 \mathrm{MeV}$ above $\varepsilon_{g}$, the groundstate band is solidly prolate. If the splitting is increased by $0.5 \mathrm{MeV}$ the lowest $J=0^{+}$and $2^{+}$become oblate, but the lowest $4^{+}$is prolate and nearly degenerate with its oblate counterpart. The two bands simply slide past, ignoring each other. To achieve any mixing, extreme fine tuning is required.

Things change when configuration mixing is allowed. In Table XIV, to the left, is the result at fixed $t=4$ with prolate ground state $\left(\varepsilon_{d}-\varepsilon_{g}=1.76 \mathrm{MeV}\right)$. The choice is made to present the two bands in their pure form. To the right, the 
TABLE XIV. Properties of the yrast bands of ${ }^{72} \mathrm{Kr}$ calculated in the $r_{3} g d$ space with the R3GD interaction (see text) [ $E$ 's in $\mathrm{MeV}, Q$ in $e \mathrm{fm}^{2}$, and $B(E 2) \equiv B\left(E 2: J_{i} \rightarrow J_{f x}\right)$ in $\left.e^{2} \mathrm{fm}^{4}\right]$. Bottom: first line, measured values from [45] (error bars subject to caution as explained in text); second line, $t \leqslant 4$ results boosted as explained in the text.

\begin{tabular}{lcccccccc}
\hline \hline & $t=4$ & \multicolumn{2}{c}{$B(E 2)$} & $t \leqslant 4$ & & \multicolumn{2}{c}{$B(E 2)$} \\
\hline$J_{i}$ & $E x$ & $Q_{s}$ & $J_{f 1}$ & $J_{f 2}$ & $E x$ & $Q_{s}$ & $J_{f 1}$ & $J_{f 2}$ \\
$0_{1}$ & 0.0 & & & & 0.00 & & & \\
$0_{2}$ & 0.24 & & & & 0.30 & & & \\
$2_{1}$ & 0.28 & -65 & 1089 & 6 & 0.46 & -54 & 586 & 372 \\
$2_{2}$ & 0.56 & 58 & 3 & 897 & 0.66 & 45 & 103 & 536 \\
$4_{1}$ & 0.83 & -77 & 1509 & 1 & 1.05 & -75 & 1387 & 75 \\
$4_{2}$ & 1.23 & 69 & 0 & 1286 & 1.43 & 64 & 36 & 1093 \\
$B\left(E 2: 2_{1} \rightarrow 0_{1}\right)=810(150)$, & $B\left(E 2: 4_{1} \rightarrow 2_{1}\right)=2720(550)$ \\
$t \leqslant 4 \times 1.4 ;$ & $B\left(E 2: 2_{1} \rightarrow 0_{1}\right)=740, B\left(E 2: 4_{1} \rightarrow 2_{1}\right)=1750$ \\
\hline \hline
\end{tabular}

$t \leqslant 4$ results show prolate dominance with strong groundstate mixing, using $\varepsilon_{d}-\varepsilon_{g}=2.26 \mathrm{MeV}$, which yields oblate ground state at fixed $t=4$. While $B\left(E 2: 2^{+} \rightarrow 0^{+}\right)$is halved, the $B\left(E 2: 4_{1}^{+} \rightarrow 2_{1}^{+}\right)$changes by less than $10 \%$. To estimate the effect of omitting the $s$ orbit, we redo calculations as in Table VIII: $\varepsilon_{d}=2 \mathrm{MeV}$ and no $s$ orbit, and then add $\varepsilon_{s}=3$ $\mathrm{MeV}$. The rates are boosted $15 \%$, and a further $10 \%$ may come from $e_{0}=2.1$, as suggested near the end of Sec. V A 4, for a total of $26 \%$. At the bottom of Table XIV the corresponding boosted values are compared with the observed ones.

Let us add two entries to the list of calculations quoted by Iwasaki and co-workers [45], which fall in two groups.

(i) Those that mix prolate and oblate states. They include VAMPIR [47]—which produces thorough mixing as a reassessment of oblate dominance previously predicted [23] — and the relativistic mean-field work of $\mathrm{Fu}$ et al. [48], close to the present results: strong $0_{1}^{+} \pm 0_{2}^{+}$ mixing and strong prolate dominance in $2_{1}^{+}$.

(ii) Those that predict oblate ground bands. They include Skyrme [24,49] and Gogny [50,51] (beyond) meanfield approaches and a sophisticated form [52] of the Kumar-Baranger model in two major oscillator shells [21].

In Sec. VIB it is explained why a majority of calculations privilege the oblate solution.

Digression. Gamow-Teller strength calculated with the present wave functions agrees nicely with observation.

\section{B. Monopole vs single-particle field}

Most mean-field-based calculations have single-particle spectra in which the $d$ orbit is some $5 \mathrm{MeV}$ above the $g$ one (as in Fig. 1 of Ref. [53]), i.e., some $2 \mathrm{MeV}$ above the values in Table VIII. As emphasized in Ref. [11] and Sec. II, $H_{m}$ is a strict two-body operator, but its action can be simulated by single-particle fields - provided it is understood that they vary as a function of the orbital occupancies - the "monopole drift" mostly owing to the filling of the largest $j$ orbit in a major shell [54]. In the problems studied here, the $r_{3}$ space can be viewed as frozen, but the $g d s$ orbits are subject to drift. Above ${ }^{56} \mathrm{Ni}$ the $r_{3}$ orbits are nearly degenerate and the $g d s$ ones are close to an $l \cdot s$ sequence. There is no direct experimental evidence for the position of the $d s$ orbits around $A=68$, but we can rely on the GEMOprogram [34], which accounts for the particle or hole spectra on all known double magics to within $200 \mathrm{keV}$ and confirms the $l \cdot s$ behavior with the $d$ orbit 2-3 MeV above the $g$ one, which upon filling comes closer to $r_{3}$ and becomes detached from $d s$, which move up to join their $r_{4}$ partners to form a pseudo- $L S$ scheme.

In ${ }^{72} \mathrm{Kr}$, as elsewhere, the structure of the states is unsensitive to monopole details but their energies are not, which explains why so many calculations place the prolate state too high.

\section{Potential energy surfaces in ${ }^{80} \mathrm{Zr}$}

In the comments to Table VIII it was noted that to the three states included for ${ }^{80} \mathrm{Zr}$ one should add a $10 \mathrm{p}-10 \mathrm{~h}, r_{3}^{14}(g d s)^{10}$ prolate state at about $1 \mathrm{MeV}$ with $B\left(E 2: 2^{+} \rightarrow 0^{+}\right) \approx 2100$ $e^{2} \mathrm{fm}^{4}$ and the $r_{3}^{24}$ closed shell at $0 \mathrm{MeV}$. Because the three prolate states are triaxial they will gain energy (of the order of 3-4 MeV according to Table IV) and dominate the low-lying spectrum. In the thorough study of Rodríguez and Egido with a Gogny force (Ref. [53], Fig. 3) this is very much the case. The main discrepancy with their work is in the positioning of the closed shell, which in the potential energy surface (Ref. [53], Fig. 2) comes some $4 \mathrm{MeV}$ below the deformed minima, which we attribute to underbinding of the latter owing to the monopole effect described above. Other calculations for ${ }^{80} \mathrm{Zr}$ include Refs. [23,24,55].

\section{Coexistence in ${ }^{84} \mathrm{Mo}$}

According to Refs. [23,24] the ground state of ${ }^{84}$ Mo is prolate and spherical, respectively. Table VIII suggests three candidates:

(I) a splendid axial rotor (all "platforms" filled in the ZRP diagrams in Fig. 3), expected to have a $2^{+}$well below the observed $440 \mathrm{keV}$;

(II) a splendid oblate rotor (Table $\mathrm{X}$ ) whose $2^{+}$is way too low;

(III) a triaxial rotor.

No direct information is available, but ${ }^{82} \mathrm{Zr}$, whose behavior is likely to be similar, provides a hint. Collating data from Refs. [56-58], the ground state starts with $2^{+}$at $407 \mathrm{keV}$ and $B\left(E 2: 2^{+} \rightarrow 0^{+}\right) \approx 1900 e^{2} \mathrm{fm}^{4}$, is interrupted by a definitely smaller $B\left(E 2: 4^{+} \rightarrow 2^{+}\right)$at 700-1200 $e^{2} \mathrm{fm}^{4}$, and then resumes with a fairly constant $Q_{0}$, albeit smaller than the one extracted from $B\left(E 2: 2^{+} \rightarrow 0^{+}\right)$. Our guess is prolate dominance in ${ }^{84} \mathrm{Mo}$ quenched by mixing.

\section{E. Beyond intrinsic states}

In mean-field studies, "going beyond" amounts to projecting and moving in the $\beta-\gamma$ plane. Here we do not have a potential energy surface but a space of discrete intrinsic states: The numerous local minima, revealed by Tables I and II, constitute a natural basis in which pairing will act as mixing 
agent. We do not know yet how to do the mixing, but we may call attention to a way of dealing with each individual state.

Diagonalize separately in the quasi and pseudo spaces and then recouple in the product space. There is nothing new with this "weak-coupling" idea except one thing: The quadrupole force(s) used in each space must be boosted as much as necessary to reproduce the quadrupole moments dictated by the MZ calculations.

\section{LOOKING BACK AND FORWARD}

The paper contains at least three significant results: (A) the numerical suggestion that pseudo-SU3 may behave as an exact symmetry (Table III) using the true quadrupole force rather than its pseudo counterpart; (B) the $p f$ to $r_{3}$ reduction in Sec. V A (we have seized a unique opportunity to study the projection from a very large realistic space into a sensible model one); (C) most important, the operation of the quasi-pseudo-SU3 tandem, which was shown to account for the onset of rotational motion in the rare earths, involving the $r_{4} h f p$ (proton) and $r_{5} i g d s$ (neutron) EEI spaces [3].

The formal basis of this successful estimate was not clear at the time. Now it can be ascribed to Nilsson-SU3 self-consistency that puts together the two classics in the field: the Bohr-Mottelson rotational model [13] plus Elliott's quadrupole force and SU3 symmetry [1], via a reinterpretation of the Nilsson model [2]. Equation (19) makes explicit the connections

$$
H=H_{\mathrm{sp}}-\frac{\hbar \omega \delta}{3} 2 q_{0} \equiv H_{\mathrm{sp}}-\beta \hbar \omega \kappa \frac{\left\langle 2 q_{0}\right\rangle}{\mathcal{N}^{2}} 2 q_{0} .
$$

On the left-hand side, the Nilsson problem amounts to calculating single-particle energies in the presence of a deformation $\delta$. The constraints on $\delta$ were left undefined and the earliest successful calculation of quadrupole moments relied on volume conservation [59]. It was much later that the Nilsson orbits could be associated with an energy minimization [21,60].

On the right-hand side of Eq. (19) we have summed up the self-consistent formulation - the MZ Eqs. (13) and (14)—with its built-in constraint: The input $\left\langle 2 q^{20}\right\rangle$ must coincide with the output $\left\langle 2 q^{20}\right\rangle$. The emphasis is on the quadrupole moment, not the energy: Nilsson orbits are sensitive to the central field, while " $\left\langle 2 q^{20}\right\rangle$ orbits," i.e., the ZRP diagrams in Fig. 3, are nearly constant, reflecting the underlying operation of pseudoquasi-SU3. The resulting interpretive framework explains the appearance of "closed shells" [the $(\lambda 0)$ representations], the natural prolate dominance, the importance of "triaxiality" [the $(\lambda \mu)$ representations], and the abrupt departure from the $p f$ regime beyond $A=68$.

The open task is to put the energetics on firmer ground, while trying to keep the approach simple, or, at least, computationally doable.

\section{ACKNOWLEDGMENTS}

We thank F. Recchia for an illuminating discussion on the recent important $B(E 2)$ measurements of ${ }^{72} \mathrm{Kr}$ [45]. This work is partly supported by Spanish Grants No. FPA2011-29854 from MICINN and No. SEV-2012-0249 from MINECO, Centro de Excelencia Severo Ochoa Programme.

Notes on authorship. A.P.Z. designed, wrote, and contributed to all sections. All authors contributed to Secs. V A and V C. A.P. contributed to the original formulation of the paper and to Secs. III and VI A. S.M.L. contributed to Sec. II.
[1] J. P. Elliott, Proc. R. Soc. London, Ser. A 245, 128 (1956).

[2] S. G. Nilsson, Mat. Fys. Medd. Dan. Vid. Selsk 29, 16 (1955).

[3] A. P. Zuker, J. Retamosa, A. Poves, and E. Caurier, Phys. Rev. C 52, R1741 (1995).

[4] Unless explicitly noted, all experimental information comes from http://www.nndc.bnl.gov/ensdf and http://www.nndc. bnl.gov/be2

[5] A. P. Zuker, B. Buck, and J. B. McGrory, Phys. Rev. Lett. 21, 39 (1968); A. P. Zuker, ibid. 23, 989 (1969).

[6] P. Navratil and W. E. Ormand, Phys. Rev. Lett. 88, 152502 (2002).

[7] A. P. Zuker, Phys. Rev. Lett. 90, 042502 (2003).

[8] J. Mendoza-Temis, J. Hirsch, and A. P. Zuker, Nucl. Phys. A 843, 14 (2010).

[9] T. Otsuka, T. Suzuki, J. D. Holt, A. Schwenk, and Y. Akaishi, Phys. Rev. Lett. 105, 032501 (2010)

[10] A. Schwenk and A. P. Zuker, Phys. Rev. C 74, 061302 (2006).

[11] E. Caurier, G. Martínez-Pinedo, F. Nowacki, A. Poves, and A. P. Zuker, Rev. Mod. Phys. 77, 427 (2005).

[12] C. J. Lister et al., Phys. Rev. Lett. 59, 1270 (1987).

[13] A. Bohr and B. Mottelson, Mat. Fys. Medd. Dan. Vid. Selsk 27, 16 (1953).
[14] M. Dufour and A. P. Zuker, Phys. Rev. C 54, 1641 (1996).

[15] A. Arima, M. Harvey, and K. Shimizu, Phys. Lett. B 30, 517 (1969); K. Hecht and A. Adler, Nucl. Phys. A 137, 129 (1969).

[16] G. Martínez-Pinedo, A. P. Zuker, A. Poves, and E. Caurier, Phys. Rev. C 55, 187 (1997).

[17] M. Honma, T. Otsuka, T. Mizusaki, and M. Hjorth-Jensen, Phys. Rev. C 80, 064323 (2009).

[18] G. Martínez-Pinedo and A. P. Zuker, Nilsson and self-consistent Nilsson programs (unpublished).

[19] J. Retamosa, J. M. Udias, A. Poves, and E. Moya de Guerra, Nucl. Phys. A 511, 221 (1990).

[20] H. L. Crawford et al., Phys. Rev. Lett. 110, 242701 (2013).

[21] M. Baranger and K. Kumar, Nucl. Phys. A 110, 490 (1968).

[22] K. Kaneko, M. Hasegawa, and T. Mizusaki, Phys. Rev. C 70, 051301(R) (2004).

[23] A. Petrovici, K. W. Schmid, and A. Faessler, Nucl. Phys. A 605, 290 (1996).

[24] M. Yamagami, K. Matsuyanagi, and M. Matsuo, Nucl. Phys. A 693, 579 (2001).

[25] B. Cederwall et al., Nature (London) 469, 69 (2011).

[26] A. Poves, J. Sánchez Solano, E. Caurier, and F. Nowacki, Nucl. Phys. A 694, 157 (2001). 
[27] M. Honma, T. Otsuka, B. A. Brown, and T. Mizusaki, Eur. Phys. J. A 25, 499 (2005).

[28] G. Dussel, E. Caurier, and A. P. Zuker, At. Data Nucl. Data Tables 39, 205 (1988).

[29] A. Gottardo et al., Phys. Rev. Lett. 109, 162502 (2012).

[30] E. Caurier and A. Poves (unpublished).

[31] C. E. Svensson et al., Phys. Rev. Lett. 82, 3400 (1999).

[32] K. Starosta et al., Phys. Rev. Lett. 99, 042503 (2007).

[33] A. Petrovici, K. W. Schmid, and A. Faessler, Nucl. Phys. A 710, 246 (2002).

[34] J. Duflo and A. P. Zuker, Phys. Rev. C 59, R2347 (1999); and program GEMO in amdc.in2p3.fr/web/dz.html

[35] A. Lemasson et al., Phys. Rev. C 85, 041303(R) (2012).

[36] S. J. Q. Robinson, T. Hoang, L. Zamick, A. Escuderos, and Y. Y. Sharon, Phys. Rev. C 89, 014316 (2014).

[37] C. Qi, J. Blomqvist, T. Back, B. Cederwall, A. Johnson, R. J. Liotta, and R. Wyss, Phys. Rev. C 84, 021301 (2011).

[38] S. Zerguine and P. Van Isacker, Phys. Rev. C 83, 064314 (2011).

[39] A. Poves and A. Zuker, Phys. Rep. 70, 235 (1981).

[40] N. Singh et al., Phys. Rev. Lett. 107, 172502 (2011).

[41] C. B. Hinke et al., Nature (London) 486, 341 (2012).

[42] G. Guastalla et al., Phys. Rev. Lett. 110, 172501 (2013).

[43] A. Gade et al., Phys. Rev. Lett. 95, 022502 (2005).

[44] P. Sarriguren, Phys. Rev. C 79, 044315 (2009).
[45] H. Iwasaki et al., Phys. Rev. Lett. 112, 142502 (2014).

[46] S. M. Lenzi, F. Nowacki, A. Poves, and K. Sieja, Phys. Rev. C 82, 054301 (2010).

[47] A. Petrovici, K. W. Schmid, and A. Faessler, Nucl. Phys. A 665, 333 (2000).

[48] Y. Fu, H. Mei, J. Xiang, Z. P. Li, J. M. Yao, and J. Meng, Phys. Rev. C 87, 054305 (2013).

[49] M. Bender, P. Bonche, and P.-H. Heenen, Phys. Rev. C 74, 024312 (2006).

[50] M. Girod, J.-P. Delaroche, A. Görgen, and A. Obertelli, Phys. Lett. B 676, 39 (2009).

[51] T. R. Rodríguez, Phys. Rev. C 90, 034306 (2014).

[52] K. Sato and N. Hinohara, Nucl. Phys. A 849, 53 (2011).

[53] T. R. Rodríguez and J. L. Egido, Phys. Lett. 705, 255 (2011).

[54] A. P. Zuker, Nucl. Phys. A 576, 65 (1994).

[55] S. J. Zheng, F. R. Xu, S. F. Shen, H. L. Liu, R. Wyss, and Y. P. Yan, Phys. Rev. C 90, 064309 (2014)

[56] A. A. Chishti et al., Phys. Rev. C 48, 2607 (1993).

[57] S. D. Paul, H. C. Jain, and J. A. Sheikh, Phys. Rev. C 55, 1563 (1997).

[58] D. Rudolph et al., Phys. Rev. C 56, 98 (1997).

[59] B. Mottelson and S. G. Nilsson, Mat. Fys. Skr. Dan. Vid. Selsk 1 (1959): No-8.

[60] V. M. Strutinsky, Nucl. Phys. A 122, 1 (1968). 